\title{
ARTICLE
}

Immunotherapy

\section{Targeting LAG3/GAL-3 to overcome immunosuppression and enhance anti-tumor immune responses in multiple myeloma}

\author{
Jooeun Bae $\mathbb{1}^{1,2} \cdot$ Fabrizio Accardi ${ }^{1,2} \cdot$ Teru Hideshima ${ }^{1,2} \cdot$ Yu-Tzu Tai $\mathbb{1}^{1,2} \cdot$ Rao Prabhala ${ }^{1,2} \cdot$ Aaron Shambley ${ }^{1,2} \cdot$ \\ Kenneth Wen ${ }^{1,2} \cdot$ Sean Rowell ${ }^{1,2} \cdot$ Paul G. Richardson $\mathbb{D}^{1,2} \cdot$ Nikhil C. Munshi $\mathbb{D}^{1,2} \cdot$ Kenneth C. Anderson $\mathbb{D}^{1,2}$
}

Received: 3 November 2020 / Revised: 29 April 2021 / Accepted: 13 May 2021 / Published online: 21 July 2021

(c) The Author(s) 2021. This article is published with open access

\begin{abstract}
Immune profiling in patients with monoclonal gammopathy of undetermined significance (MGUS), smoldering multiple myeloma (SMM), and multiple myeloma (MM) provides the framework for developing novel immunotherapeutic strategies. Here, we demonstrate decreased $\mathrm{CD}^{+}{ }^{+} \mathrm{Th}$ cells, increased Treg and G-type MDSC, and upregulation of immune checkpoints on effector/regulatory and $\mathrm{CD} 138^{+}$cells in MM patients, compared MGUS/SMM patients or healthy individuals. Among the checkpoints profiled, LAG3 was most highly expressed on proliferating $\mathrm{CD} 4^{+} \mathrm{Th}$ and $\mathrm{CD} 8^{+} \mathrm{Tc}$ cells in MM patients BMMC and PBMC. Treatment with antibody targeting LAG3 significantly enhanced T cells proliferation and activities against MM. XBP1/CD138/CS1-specific CTL generated in vitro displayed anti-MM activity, which was further enhanced following anti-LAG3 treatment, within the antigen-specific memory T cells. Treg and G-type MDSC weakly express LAG3 and were minimally impacted by anti-LAG3. CD138 ${ }^{+}$MM cells express GAL-3, a ligand for LAG3, and anti-GAL-3 treatment increased MM-specific responses, as observed for anti-LAG3. Finally, we demonstrate checkpoint inhibitor treatment evokes non-targeted checkpoints as a cause of resistance and propose combination therapeutic strategies to overcome this resistance. These studies identify and validate blockade of LAG3/GAL-3, alone or in combination with immune strategies including XBP1/CD138/CS1 multipeptide vaccination, to enhance anti-tumor responses and improve patient outcome in MM.
\end{abstract}

\section{Introduction}

Despite major improvements in the treatment of multiple myeloma (MM), novel agents targeting the tumor and its microenvironment are urgently needed. The reciprocal interaction between $\mathrm{MM}$ and bone marrow (BM) accessory cells induces genomic, epigenomic, and functional changes, which in turn promote tumor development and progression, cell adhesion mediated-drug resistance, and immunosuppression. In prior studies, we and others have delineated mechanisms and sequelae of interactions among

Supplementary information The online version contains supplementary material available at https://doi.org/10.1038/s41375021-01301-6.

$\triangle$ Jooeun Bae

jooeun_bae@dfci.harvard.edu

1 Dana-Farber Cancer Institute, Boston, MA, USA

2 Harvard Medical School, Boston, MA, USA
MM, stromal, and accessory cells [1-3]. These studies have enhanced our understanding of MM pathogenesis, delineated changes within tumor cells and the BM milieu underlying progression from monoclonal gammopathy of undetermined significance (MGUS) to smoldering MM (SMM) to active MM, and provided the framework for overcoming immunosuppression in the BM milieu.

Immunotherapeutic approaches to develop tumorspecific memory $\mathrm{T}$ cells and overcome inhibitory mechanisms in the tumor microenvironment have the potential to achieve prolonged anti-tumor immune and clinical responses. Currently, one of the most effective therapeutic strategies modulates immune checkpoints, which regulate the balance between immune response and tolerance [4, 5]. Targeting immune suppressive cells, blocking inhibitory molecules on suppressive/regulatory and tumor cells, and activating costimulatory molecules on effector cells also represent promising therapeutic approaches to enhance anti-tumor immunity and improve therapeutic efficacy. In MM, PD1 blockade in combination with either pomalidomide or lenalidomide was associated 
with adverse outcome, which has limited evaluation of other immune modulators (checkpoint inhibitors) in the clinic. A better understanding of the immunologic effects of checkpoint inhibitors and immune agonists in the BM tumor microenvironment would further delineate their role in pathogenesis and inform their therapeutic application, alone or in combination with other immunotherapies.

Cancer vaccines have been shown to generate antigenspecific effector $\mathrm{T}$ cells against tumor with a favorable therapeutic index [6-8]. Importantly, cancer vaccine therapies have the potential to develop memory $\mathrm{CD}^{+}$ CTL specifically directed at selected tumor-associated antigens and induce long-term anti-tumor immunity $[9,10]$. Although vaccines induce antigen-specific memory $\mathrm{CD}^{+}$CTL expressing costimulatory (CD28, 41BB) and activation (CD38, CD69) markers, these memory CTL also upregulate various inhibitory checkpoints (CTLA4, PD1, LAG3, TIM3, VISTA), which may in turn abrogate their function [11-13]. Previously, in preclinical and clinical studies, we reported that XBP1/CD138/CS1 multipeptide can induce antigen-specific memory CTL against MM [14-18], and that this therapeutic approach when combined with optimal immune modulators may further overcome immunosuppression characteristic of $\mathrm{MM}$ $[19,20]$.

In the current studies, we first investigated the potential impact of checkpoint inhibitors and immune agonists on effector $\mathrm{T}$ cells, accessory cells, and regulatory cells in MM. These studies demonstrate increased immune suppressor cells and upregulated inhibitory checkpoint molecules in patients with MM patients (newly diagnosed, relapsed, relapsed/refractory) compared to premalignant disease patient (MGUS, SMM). We show that blocking immune checkpoints (PD1, LAG3), alone and in combination, can enhance effector T-cell responses in the tumor microenvironment of MM patients to a greater extent than by stimulating costimulatory molecules (OX40, GITR). Importantly, we demonstrated increased LAG3 expression on proliferating $\mathrm{CD}^{+} \mathrm{T}$ cells in MM patient BMMC and PBMC, as well as robust surface and intracellular expression of its ligand, GAL- 3 , in CD138 ${ }^{+}$patient MM cells and MM cell lines. Moreover, LAG3/GAL-3 blockade can efficiently enhance the proliferation of $\mathrm{T}$ cells in $\mathrm{MM}$ patients and functional activities of MM-specific CTL, including XBP1/CD138/CS1-targeting memory $\mathrm{CD}^{+}$ $\mathrm{T}$ cells, against MM. These studies provide the rationale for inhibiting LAG3/GAL-3, in combination with immunotherapy including a cancer vaccine, to overcome the immune suppressive tumor microenvironment, enhance anti-tumor-specific immune responses, and improve patient outcome in MM.

\section{Methods}

\section{Cell lines and preparation of tumor cell lysates or irradiated whole tumor cells}

MM cell lines U266, McCAR, HSB2, MM1S, RPMI8226, OPM2, H929, ANBL6, OCIMY5, AMO1, and KMS11 were obtained from ATCC (Manassas, VA). The T2 cell line, a human B- and T-cell hybrid expressing HLA-A2 molecules, was provided by Dr J. Molldrem (University of Texas M. D. Anderson Cancer Center, Houston, TX). The cell lines were cultured in DMEM media supplemented with $10 \%$ fetal calf serum (BioWhittaker, Walkersville, MD), $100 \mathrm{IU} / \mathrm{ml}$ penicillin, and $100 \mu \mathrm{g} / \mathrm{ml}$ streptomycin (GibcoLife Technologies, Rockville, MD). A mixture of ten MM cell lines was utilized to prepare tumor cell lysates by repeated $(10 \mathrm{X})$ cycles of freeze $\left(-140^{\circ} \mathrm{C}\right)$ /thaw $\left(37^{\circ} \mathrm{C}\right)$ or prepared as irradiated $(20 \mathrm{~Gy})$ whole tumor cells as sources of MM antigen stimulation.

\section{Reagents}

Fluorochrome-conjugated anti-human monoclonal antibodies (mAb) specific to CD3, CD4, CD8, CD11b, CD11b, CD14, CD15, CD25, CD28, CD33, CD38, CD69, CD138, FOXP3, HLA-DR, PD1, PD-L1, PD-L2, CTLA4, LAG3, TIM3, VISTA, ICOS, OX40, GITR, GAL-3, GAL-9, ICOS-L, HLA-DP/DQ/DR, CCR7, CD45RO, CD69, CD107a, or IFN- $\gamma$ were purchased from Becton Dickinson (BD) (San Diego, CA), LifeSpan Biosciences (Seattle, WA), BioLegend (San Diego, CA), or eBioscience (San Diego, CA). Live/Dead Aqua Fixable Cell Stain Kit was purchased from Molecular Probes (Grand Island, NY). Recombinant human GM-CSF was obtained from Immunex (Seattle, WA), and human IL-2, IL-4, IFN- $\alpha$, and TNF- $\alpha$ were purchased from R\&D Systems (Minneapolis, MN). Clinical grade checkpoint inhibitor (anti-PD1, anti-LAG3) or immune agonist (anti-OX40, anti-GITR) were provided by Bristol Myers Squibb (New York, NY).

\section{BM or peripheral blood (PB) samples from MGUS, SMM, or MM patients or healthy donors}

$\mathrm{BM}$ aspirates and $\mathrm{PB}$ samples were obtained from patients with MGUS [BM: $N=5$, PB: $N=5]$ and SMM [BM: $N=$ 5, PB: $N=5$ ] and patients with $\mathrm{MM}$ (newly diagnosed [BM: $N=18$, PB: $N=10]$, relapsed [BM: $N=14$, PB: $N=$ 12], relapsed/refractory [BM: $N=18$, PB: $N=12]$ ) after informed consent, in accordance with the Declaration of Helsinki, and with approval by the Institutional Review Board at Dana-Farber Cancer Institute (Boston, MA). In 
addition, healthy individuals $\mathrm{BM}[N=5]$ or leukapheresis $[N=12]$ products were purchased from either AllCells (Alameda, CA) or the Blood Donor Center at Boston Children's Hospital (Boston, MA), respectively. Mononuclear cells were isolated from BM (BMMC) or PB (PBMC) by standard density gradient centrifugation using Ficoll-Paque $^{\mathrm{TM}}$ Plus (Amersham Pharmacia Biotech AB, Uppsala Sweden) and used in these studies.

\section{Phenotypic characterization of immune and regulatory cell subsets and expression of checkpoints or costimulatory molecules}

BMMC or PBMC from patients with MGUS, SMM, $\mathrm{MM}$, or healthy individuals were evaluated by flow cytometry analyses by staining cells with fluorochromeconjugated mAb specific to each cell surface antigen for $30 \mathrm{~min}$ at room temperature, followed by LIVE/DEAD reagent staining to confirm viability. Regulatory $T$ cells (Treg) were identified by cell surface staining (CD3, CD4, CD8, CD25), permeabilized using Foxp3/Transcription Factor Staining Buffer Set (eBioscience) and stained for intracellular FOXP3 expression. Myeloidderived suppressor cells (MDSC) were identified as Gtype MDSC $\left(\mathrm{CD} 11 \mathrm{~b}^{+} \mathrm{CD}^{+} 3^{+}\right.$HLA-DR $^{\text {low/- }} \mathrm{CD} 14^{-}$ $\left.\mathrm{CD} 15^{+}\right)$and M-type MDSC $\left(\mathrm{CD} 11 \mathrm{~b}^{+} \mathrm{CD} 3^{+} \mathrm{HLA}^{-}\right.$ $\left.\mathrm{DR}^{\text {low/- }} \mathrm{CD} 14^{+} \mathrm{CD} 15^{-}\right)$. Cells were acquired using a BD Fortessa X-20 (BD Biosciences) flow cytometer, and the data were analyzed using DIVA ${ }^{\mathrm{TM}}$ v8.0 (BD) or FlowJo v10.0.7 (Tree star, Ashland, OR) software.

\section{Generation of MM-specific CTL ex vivo with immunogenic XBP1/CD138/CS1 peptides}

XBP1/CD138/CS1-specific CTL were generated ex vivo after

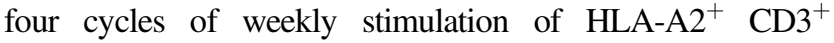
T lymphocytes $(N=5)$ with a cocktail of four peptides containing heteroclitic XBP1 US $184-192$ (YISPWILAV), heteroclitic XBP1 $\mathrm{SP}_{367-375}$ (YLFPQLISV), native CD138 $260-268$ (GLVGLIFAV), and native $\mathrm{CS}_{239-247}$ (SLFVLGLFL), as described previously [14-17].

\section{Cell proliferation by carboxy fluorescein succinimidyl ester (CFSE) tracking}

Proliferation of specific cell populations was evaluated using CFSE-based proliferation assays $(N=5)$. In brief, MM patient BMMC, PBMC, or XBP1/CD138/CS1specific CTL were labeled with CFSE (Molecular
Probes, Eugene, OR) and incubated with clinical grade checkpoint inhibitor $(1 \mu \mathrm{g} / \mathrm{ml})$ or immune agonist $(1 \mu \mathrm{g} /$ $\mathrm{ml}$ ) in the presence of low dose (20 units) IL-2, with or without stimulation with irradiated MM cells (patients' cells, cell line) or MM lysates. After 4-7 days incubation, the cells were stained with LIVE/DEAD reagent and specific fluorochrome-conjugated mAbs, washed, fixed in $2 \%$ paraformaldehyde, and acquired using a LSRII Fortessa ${ }^{\mathrm{TM}}$ flow cytometer.

\section{XBP1/CD138/CS1-specific CTL functional activities measured by CD107a degranulation and intracellular IFN- $\gamma$ production}

The anti-tumor activities of MM-specific CTL were measured by $\mathrm{CD} 107 \mathrm{a}$ degranulation and IFN- $\gamma$ production against MM $(N=5)$. In brief, XBP1/CD138/CS1-specific CTL were treated with clinical grade anti-PD1 or antiLAG3 $(1 \mu \mathrm{g} / \mathrm{ml})$ for $24 \mathrm{~h}$. The cells were then cultured with U266 MM cells in the presence of CD107a mAb. After 1-h incubation, brefeldin A (BD) and monensin (BD) were added, and cultures were incubated for an additional $5 \mathrm{~h}$. Cells were harvested, washed in PBS, stained with LIVE/ DEAD reagent, washed, incubated with fluorochromeconjugated $\mathrm{mAb}$ to identify $\mathrm{T}$ cells, allowing for assays of their functional activity against MM. After surface staining, cells were fixed/permeabilized, stained for Th1 cytokines, washed with Perm/Wash solution (BD), and analyzed by flow cytometry. Multipeptide-CTL specific CD107a degranulation and Th1 cytokine production were analyzed using DIVA ${ }^{\mathrm{TM}}$ v8.0 or FlowJo v10.0.7 software.

\section{Statistical analysis}

Results are presented as mean \pm standard error. Groups were compared using an unpaired Student's $t$ test. Differences were considered significant when $p<0.05$.

\section{Results}

\section{Impact of clinical grade immune modulator treatment on proliferation of MM patient $\mathrm{T}$ cells expressing checkpoint or costimulatory molecules}

We first evaluated proliferation to low dose IL-2 in specific T-cell subsets in BMMC or PBMC from patients with newly diagnosed, relapsed, or relapsed/refractory MM using CFSE-based assays. Proliferation of $\mathrm{CD}^{+} \mathrm{T}$ cells expressing PD1, LAG3, OX40, or GITR was significantly $\left({ }^{*} p<\right.$ 0.05 ) higher as compared to total $\mathrm{CD}^{+} \mathrm{T}$ cells in $\mathrm{MM}$ 
Fig. 1 Characterization of checkpoint expression on MM patient $\mathbf{T}$ cells. BMMC from MM patients (newly diagnosed, relapsed, relapsed/refractory) were treated with low dose (20 units) IL-2 and evaluated for proliferation of specific T-cell subsets in CFSE assay. A CD3 ${ }^{+}$ T cells expressing PD1, LAG3, OX40, or GITR had significantly $(* p<0.05)$ higher proliferation compared to total $\mathrm{CD}^{+} \mathrm{T}$ cells in cultures of BMMC from MM patients $(N=$ $10)$, with the highest expansion of T-cell subsets expressing LAG3. B. Treatment of BMMC from MM patients $(N=10)$ with clinical grade anti-PD1, antiLAG3, or anti-OX40 enhanced proliferation of $\mathrm{T}$ cells in both $\mathrm{CD} 4^{+}$Th cells and $\mathrm{CD} 8^{+} \mathrm{Tc}$ cells, with the highest $(* p<$ 0.05 ) increase with anti-LAG3 treatment. C Treatment of newly diagnosed $(N=6)$ or relapsed $(N=3)$ MM patients PBMC with clinical grade anti-LAG3 or anti-OX40 enhanced proliferation of $\mathrm{CD}^{+} \mathrm{T}$ cells, with the highest $(* p<0.05)$ increase with anti-LAG3 treatment, with or without MM lysates stimulation.
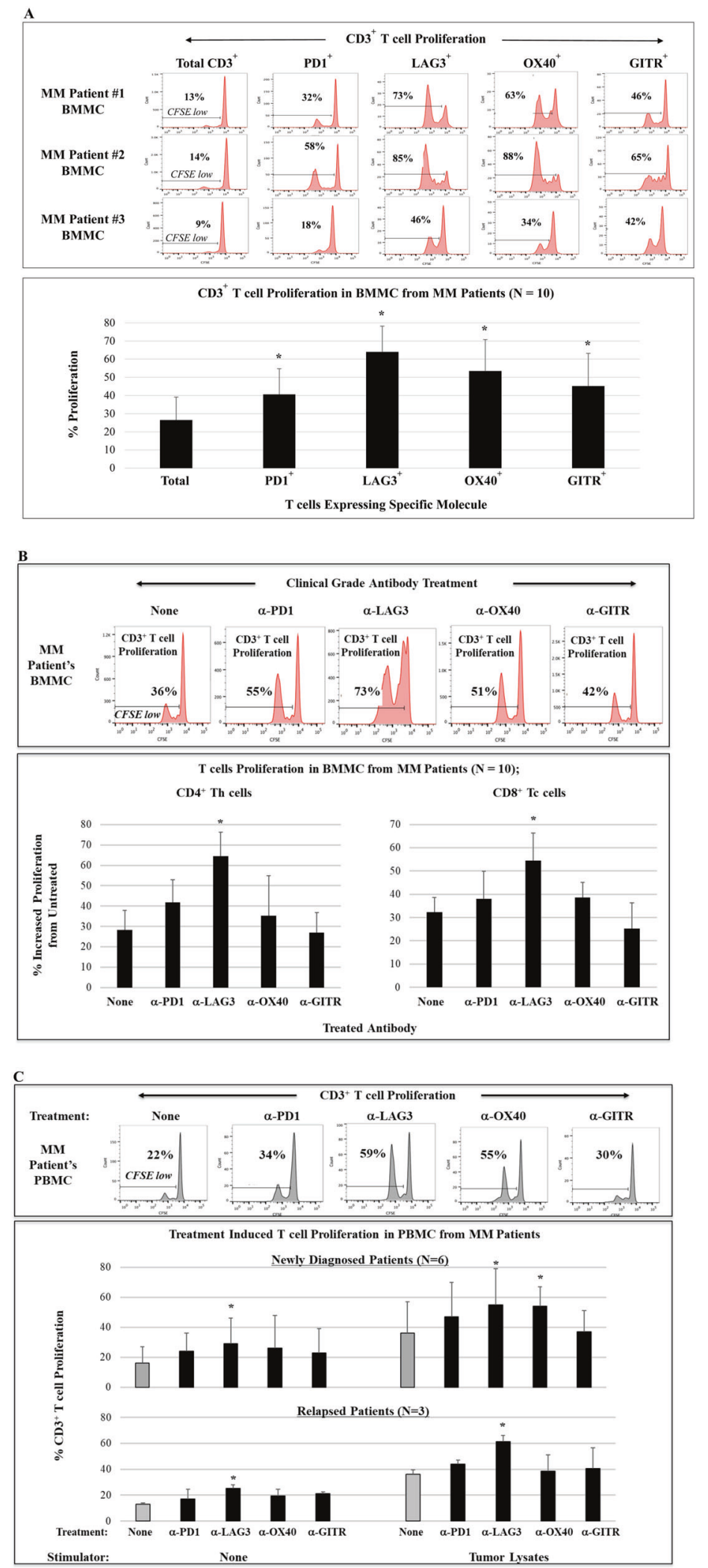

patient BMMC. In BMMC from patients $(N=10)$ with newly diagnosed, relapsed, or relapsed/refractory MM, Tcell subsets expressing the LAG3 immune checkpoint demonstrated the highest $\left({ }^{*} p<0.05\right)$ proliferation (Fig. 1A; histograms, bar graph). We next assessed the impact of clinical grade immune modulators on T-cell proliferation 
within BMMC from patients with newly diagnosed, relapsed, or relapsed/refractory $\mathrm{MM}(N=10)$. Overall, $\mathrm{CD}^{+}{ }^{+}$T-cell proliferation was triggered by treatment with each clinical grade antibody (PD1, LAG3, OX40, GITR) compared to untreated control (Fig. 1B). Of note, a significant increase $(* p<0.05)$ in proliferation of $\mathrm{CD}^{+} \mathrm{Th}$ cells was induced by treatment with anti-PD1 or anti-LAG3, and increased $(* p<0.05)$ proliferation of $\mathrm{CD}^{+}$Tc cells after treatment with anti-LAG3 or anti-OX40. The highest proliferation in both $\mathrm{CD} 4^{+} \mathrm{Th}$ cells and $\mathrm{CD} 8^{+} \mathrm{Tc}$ cells was induced by anti-LAG3 treatment (histograms, bar graphs). Next, MM patient T-cell proliferation in response to tumor lysates from ten different MM cell lines, in the presence or absence of immune modulator, was examined. As shown in Fig. 1C, PBMC from patients with newly diagnosed $(N=6)$ or relapsed $(N=3) \mathrm{MM}$ treated with anti-LAG3 had significantly $(* p<0.05)$ higher $\mathrm{T}$-cell proliferation than with the other clinical grade immune modulators anti-PD1, antiOX40, and anti-GITR, either in the presence or absence of MM lysate stimulation. In addition, BMMC from MM patients $(N=5)$ treated with anti-LAG3 had significantly higher $(* p<0.05)$ T-cell proliferation than with the other clinical grade immune modulators, upon stimulation with the mixture of ten different MM cell lines, either as irradiated whole cells or tumor lysates. The tumor lysates induced a greater T-cell response in MM patients' BMMC $(N=5)$ than irradiated whole tumor cells, which was enhanced to a greater extent by checkpoint inhibitors (antiLAG3 > anti-PD1) than by immune agonists (anti-OX40, anti-GITR) (Supplementary Fig. 1). Taken together, these data indicate the therapeutic potential of LAG3 blockade to effectively augment $\mathrm{T}$-cell proliferation directed against MM.

\section{Decreased effector CD4 ${ }^{+}$Th cells, increased regulatory and immune suppressor cells, and upregulation of immune checkpoints in active MM patients compared to MGUS/SMM patients or healthy donors}

We and others have characterized the impact of interactions among tumor, stromal, and accessory cells on MM cell growth, survival, and drug resistance [1-3]. We therefore next evaluated immune effector, regulatory/suppressor, and tumor cells for expression of key immune checkpoints using freshly isolated BMMC and PBMC from patients with MGUS, SMM, or MM, as well as normal healthy individuals. Compared to MGUS/SMM patients or healthy individuals, MM patients' (newly diagnosed, relapsed, relapsed/refractory) BMMC and PBMC had significantly $\left({ }^{*} p<0.05\right)$ decreased $\mathrm{CD} 4^{+} \mathrm{Th}$, but not $\mathrm{CD}^{+}$Tc cells (data not shown), as well as increased $\mathrm{CD}^{+}$Treg $\left(\mathrm{CD}^{+} \mathrm{CD}^{+} / \mathrm{FOXP}^{+}{ }^{+} \mathrm{CD} 25^{+}\right.$) (Fig. $\left.2 \mathrm{~A}\right)$. Of note, PD1 was more highly expressed on $\mathrm{CD} 4^{+}$Treg within BMMC from MM patients than patients with MGUS/SMM or healthy individuals and demonstrated higher expression of PD1 than LAG3 or GITR (Fig. 2B). We next showed that G-type $\operatorname{MDSC}\left(\mathrm{CD}_{11 b^{+}} \mathrm{CD}_{3}{ }^{+} \mathrm{HLA}_{-D R}{ }^{\text {low/- }} \mathrm{CD}^{-} 4^{-} \mathrm{CD} 15^{+}\right)$, but not M-type MDSC $\left(\mathrm{CD}_{11 b^{+}} \mathrm{CD}^{+} 3^{+}\right.$HLA-DR $^{\text {low/- }}$ $\left.\mathrm{CD} 14^{+} \mathrm{CD} 15^{-}\right)$, are significantly $(* p<0.05)$ increased in BMMC of MM patients (highest in relapsed/refractory MM) compared to MGUS/SMM patients or healthy donors (Fig. 3A). Moreover, G-type MDSC in BMMC and PBMC of MM patients expressed significantly higher levels of PDL1 than PD-L2 or LAG3 (Fig. 3B). Finally, CD138 ${ }^{+}$MM cells (newly diagnosed, relapsed, relapsed/refractory) had significantly $(* p<0.05)$ higher expression of PD-L1, but not PD-L2, than healthy donors (Fig. 3C). In addition, $\mathrm{CD}^{+}$Th cells and $\mathrm{CD}^{+}$Tc cells in MM patients had significantly higher PD1 expression compared to MGUS/ SMM patients or healthy individuals (data not shown). Thus, these studies reveal heterogeneity in the proportion of immune cell subsets among patients with MGUS, SMM, or MM, and healthy individuals; decreased effector cells, increased Treg, and G-type MDSC, as well as upregulation of immune checkpoints on effector, regulatory, and $\mathrm{CD} 138^{+} \mathrm{MM}$ cells are observed in MM patients compared to patients with MGUS or SMM or healthy individuals.

\section{Higher intracellular than surface expression of immune checkpoints in MM patient BM}

To better understand potential mechanisms of resistance to checkpoint inhibitor therapy in MM, we next examined the distribution and localization (cell surface vs. intracellular) of key immune checkpoints in BMMC from MM patients $(N$ $=9)$. $\mathrm{CD}^{+} \mathrm{T}$ cells more highly expressed PD1 and LAG3 than CTLA4 and TIM3, with significantly $\left({ }^{*} p<0.05\right)$ greater intracellular CTLA4, PD1, and LAG3 expression than cell surface levels (Fig. 4A; histograms, bar graph). In contrast, surface and intracellular expression levels of TIM3 were similar. On the CD138 $8^{+}$MM cells, GAL-9 and ICOSL were more highly expressed than PD-L1 and PD-L2, with higher intracellular than cell surface expression of PD-L1, PD-L2, GAL-9, and ICOS-L (Fig. 4B; histograms, bar graph). These results support an extended treatment protocol with checkpoint inhibitors to overcome the high intracellular reservoir of immune checkpoints, and thereby overcome immunosuppression and improve outcome in MM.

\section{Induction of another checkpoint expression and Treg triggered by treatment with checkpoint inhibitor or immune agonist}

To further elucidate potential mechanisms of resistance to checkpoint inhibitor or immune agonist therapy in MM 
Fig. 2 Characterization of regulatory $T$ cells in BMMC or PBMC from patients with MGUS, SMM, or MM and healthy individuals. Freshly isolated BMMC or PBMC from patients with MGUS (BM: $N=5$, PB: $N=5$ ), SMM (BM: $N=5$, PB: $N=5$ ), newly diagnosed MM (BM: $N=5$, PB: $N=5$ ), relapsed MM (BM: $N=5$, PB: $N=5)$, relapsed/refractory MM (BM: $N=5$, PB: $N=5$ ), and healthy donors (BM: $N=4$, PB: $N=5$ ) were evaluated for the frequency of regulatory $\mathrm{T}$ cells and their expression of immune checkpoints. A MM patients (newly diagnosed, relapsed, relapsed/refractory) had a significantly $(* p<0.05)$ higher $\mathrm{CD}^{+}$Treg $\left(\mathrm{CD} 25^{+} \mathrm{FOXP}^{+} / \mathrm{CD}^{+} \mathrm{CD}^{+}\right)$in BMMC and PBMC compared to MGUS patients, SMM patients or healthy individuals. B CD4 ${ }^{+}$ Treg cells from active MM patients had significantly $\left({ }^{*} p<0.05\right)$ higher PD1, but not LAG3 or GITR, expression in BMMC as compared to MGUS/ SMM patients or healthy individuals.
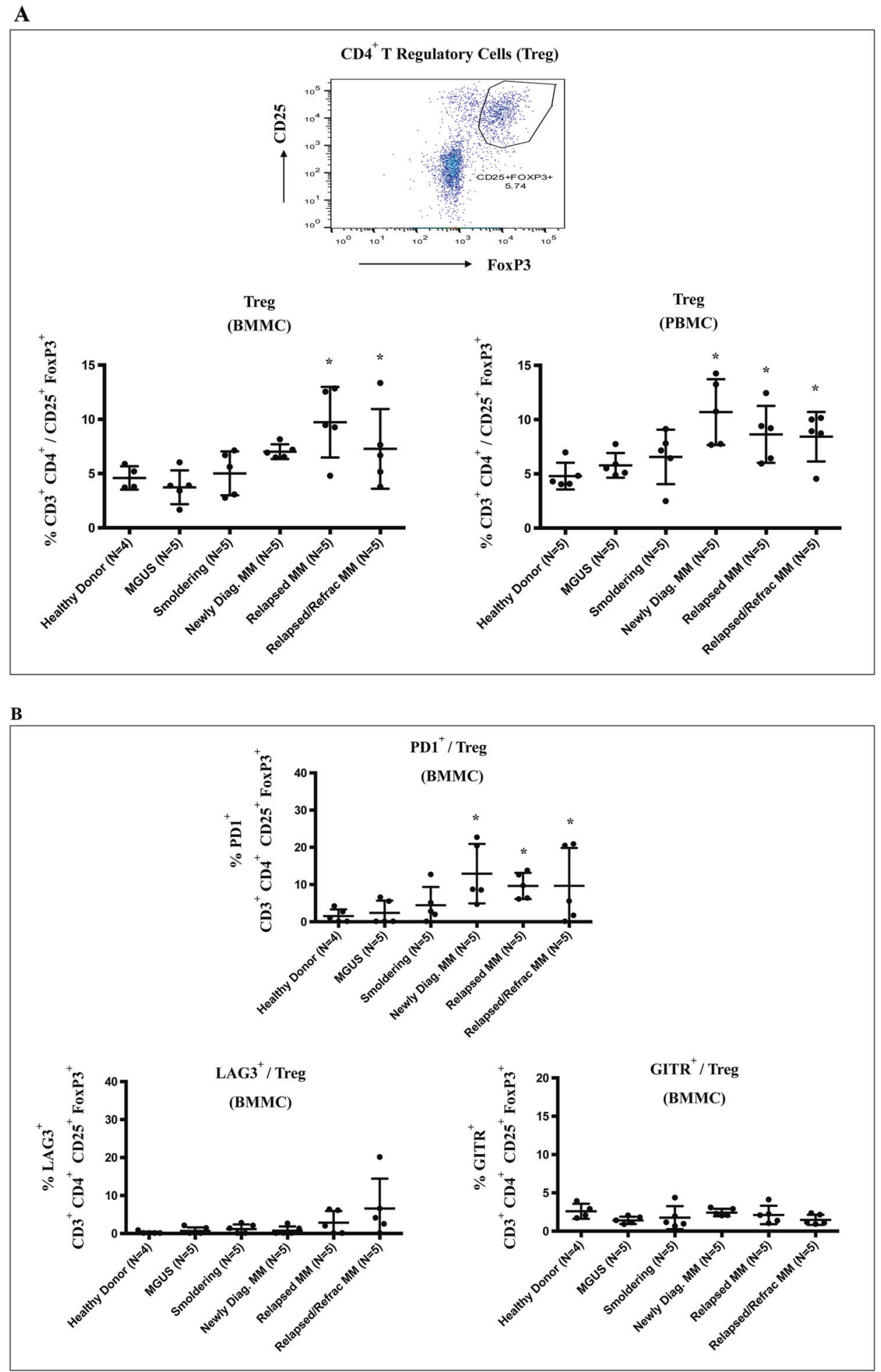

patients, we next evaluated the effects of clinical grade modulators on effector and Treg cell subsets in tumor microenvironment. Importantly, treatment of MM patients' $(N=10)$ BMMC with the specific mAb targeting PD1, LAG3, OX40, or GITR induced upregulation of PD1 and
LAG3 expression on T cells (Fig. 5A; histograms, bar graph). Of note, anti-PD1 triggered proliferation of T cells expressing an alternative immune checkpoint to a greater extent than anti-LAG3 treatment. Moreover, checkpoint inhibitor or immune agonist treatment of MM patient' $(N=5)$ BMMC 
Fig. 3 Characterization of MDSC and CD138 ${ }^{+} \mathrm{MM}$ cells in BMMC or PBMC from patients with MGUS, SMM, or MM and healthy donors. Freshly isolated BMMC or PBMC from patients with MGUS (BM: $N=5$, PB: $N=5$ ), SMM (BM: $N=5, \mathrm{~PB}: N=5$ ), newly diagnosed $\mathrm{MM}$ (BM: $N=5$, PB: $N=5$ ), relapsed MM (BM: $N=5$, PB: $N=5$ ), relapsed/refractory MM (BM: $N=5$, PB: $N=5$ ), and healthy donors (BM: $N=4$, PB: $N=5$ ) were evaluated for the frequency of MDSC or $\mathrm{CD} 138^{+} \mathrm{MM}$ cells and their expression of immune checkpoints. A MM patients BMMC had significantly $\left({ }^{*} p<0.05\right)$ higher G-type MDSC $\left(\mathrm{CD} 11 \mathrm{~b}^{+} \mathrm{CD}^{+} 3^{+}\right.$HLA$\left.\mathrm{DR}^{\text {low/- }} \mathrm{CD}^{-} 4^{-} \mathrm{CD} 15^{+}\right)$, but not M-type MDSC (CD11 b

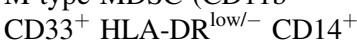
$\mathrm{CD}^{-} 5^{-}$, compared to MGUS/ SMM patients or healthy individuals. B G-type MDSC from MM patients BMMC and PBMC expressed significantly $\left({ }^{*} p<0.05\right)$ higher PD-L1, but not PD-L2 or LAG3, than MGUS and SMM patients or healthy individuals. C CD138 ${ }^{+}$ tumor cells in MM patient BMMC have significantly $(* p<0.05)$ higher PD-L1, but not PD-L2, expression as compared to BMMC from healthy individuals.
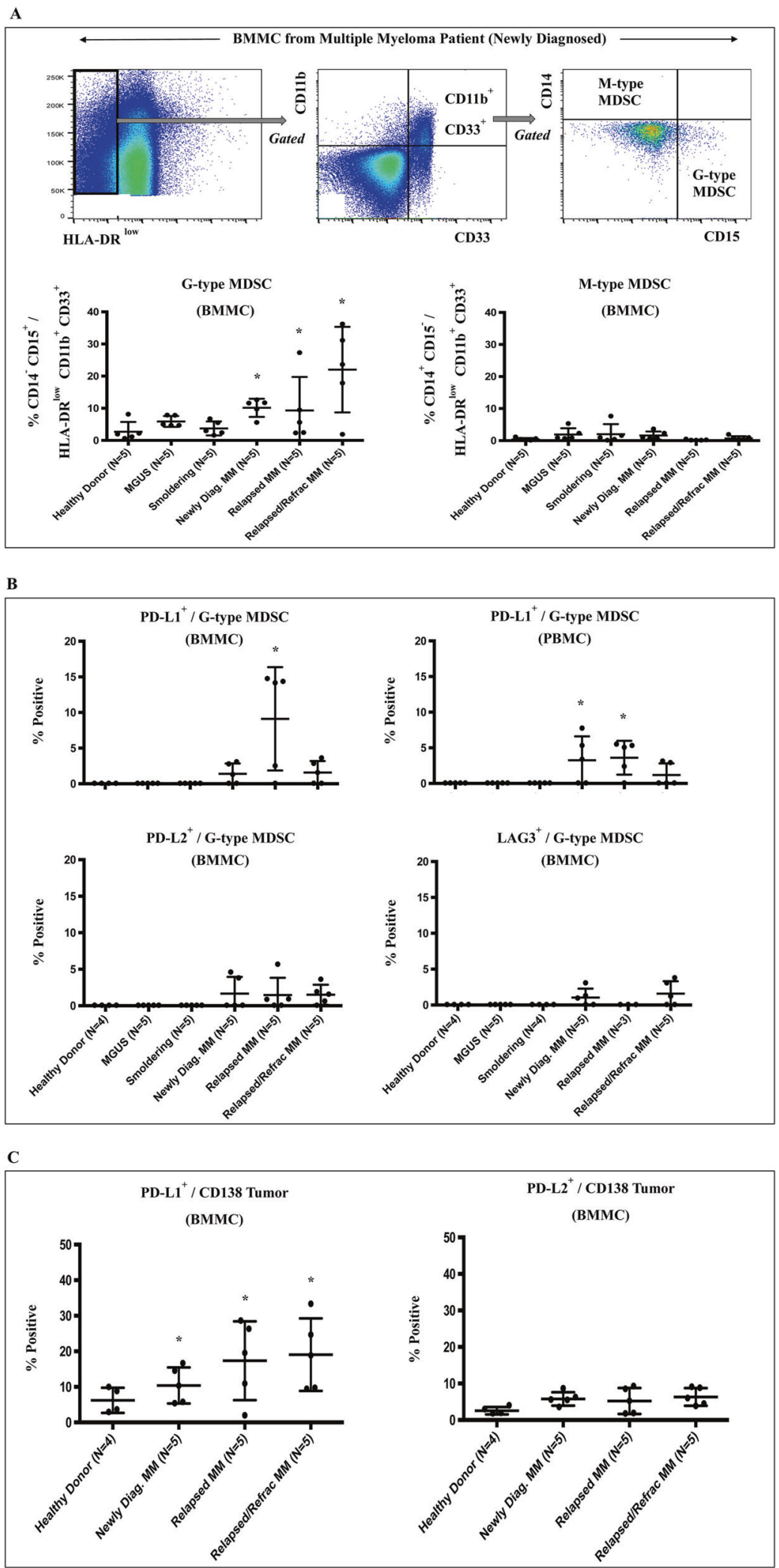
Fig. 4 Distribution, location, and expression level of key immune checkpoints, activation, and costimulatory molecules in MM patients' BMMC. Cell surface and intracellular expression of immune checkpoints were evaluated and compared in BMMC from MM patients (newly diagnosed, relapsed, relapsed/refractory; $N=9$ ) using flow cytometry. $\mathbf{A} \mathrm{CD}^{+} \mathrm{T}$ cells in BMMC from MM patients showed an increased expression of PD1 and LAG3 than CTLA4 or TIM3. Intracellular expression of CTLA4, PD1, and LAG3 was significantly $(* p<0.05)$ greater than corresponding cell surface expression levels. B Primary $\mathrm{CD}_{138^{+}}$patient MM cells showed increased cell surface expression of GAL-9/ICOS-L as compared with PD-L1/PD-L2, as well as significantly $(* p<0.05)$ higher intracellular expression of GAL-9 and ICOS$\mathrm{L}$ than cell surface expression.
A
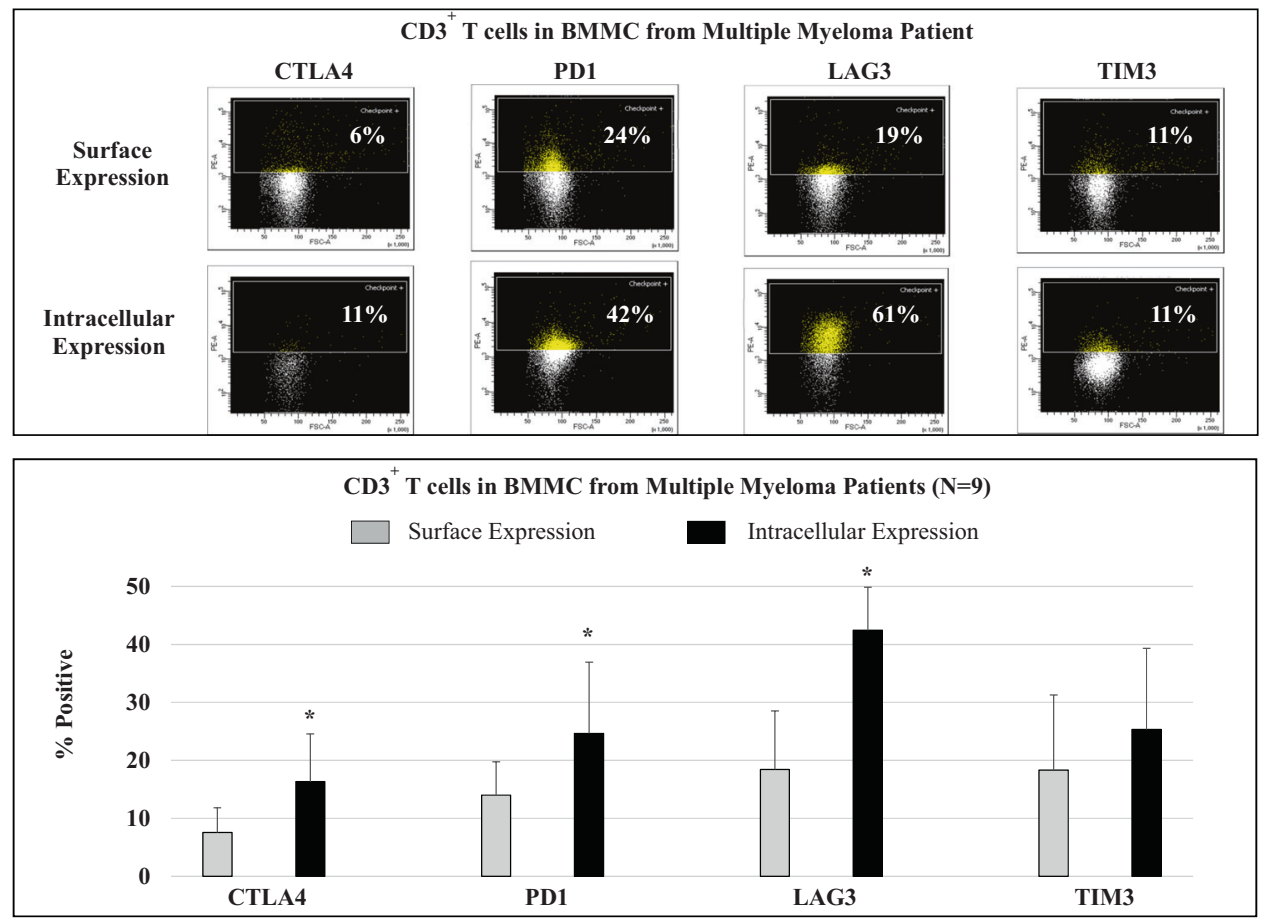

B
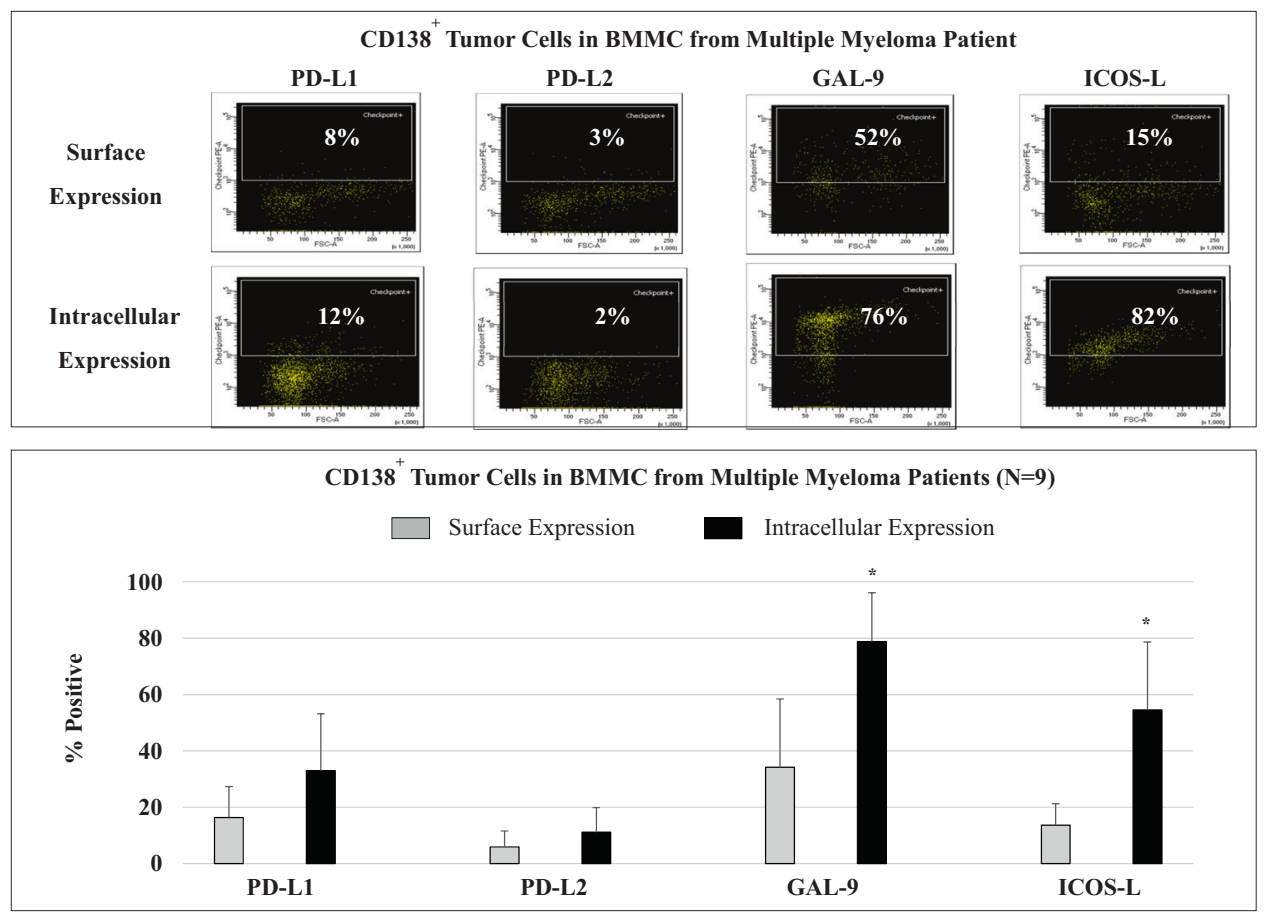

increased Treg proliferation, with a significantly $(* p<0.05)$ high induction by anti-PD1 or anti-OX40 and the lowest induction by anti-LAG3 (Fig. 5B; histograms, bar graph). We also investigated the impact of single agent or combination modulator treatment on Treg expansion in the MM microenvironment (Fig. 5C). Treatment of MM patients' $(N$ $=3$; relapsed/refractory) BMMC with single agent anti-PD1, anti-OX40, or anti-GITR triggered a significant $(* p<0.05)$ expansion of Treg. A decreased level of Treg proliferation was observed upon combination treatment with checkpoint 
Fig. 5 Impact of checkpoint inhibitor or immune agonist treatment on MM patients' BMMC. BMMC from MM

patients were treated with clinical grade checkpoint inhibitor or immune agonist in the presence of low level of IL-2 (20 units $/ \mathrm{ml}$ ) and examined for checkpoint expression and immune function. A Treatment of BMMC from MM patients $(N$ $=10$ ) with anti-PD1, antiLAG3, anti-OX40, or anti-GITR increased expansion of $\mathrm{T}$ cells expressing another immune checkpoint. B Treatment of BMMC of MM patients $(N=5)$ induced $\mathrm{CD}^{+}{ }^{+}$Treg proliferation, with the highest increase triggered by anti-PD1 $(* p<0.05)$ and the lowest by anti-LAG3. C Treatment of BMMC from MM patients $(N=$ 3) with single agent anti-PD1, anti-OX40, or anti-GITR enhanced $(* p<0.05)$ proliferation of Treg, which was decreased by combination treatment with checkpoint inhibitor $(\alpha-\mathrm{PD} 1+\alpha$-LAG3) compared to combination with immune agonist $(\alpha-\mathrm{OX} 40+\alpha$ GITR) or single agent treatment.

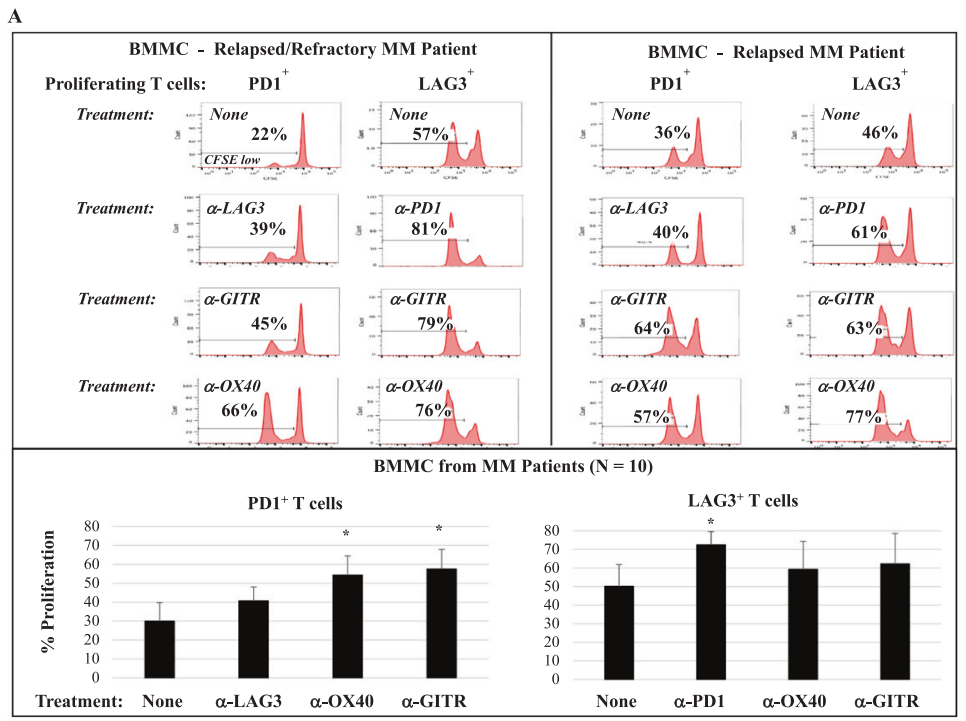

B
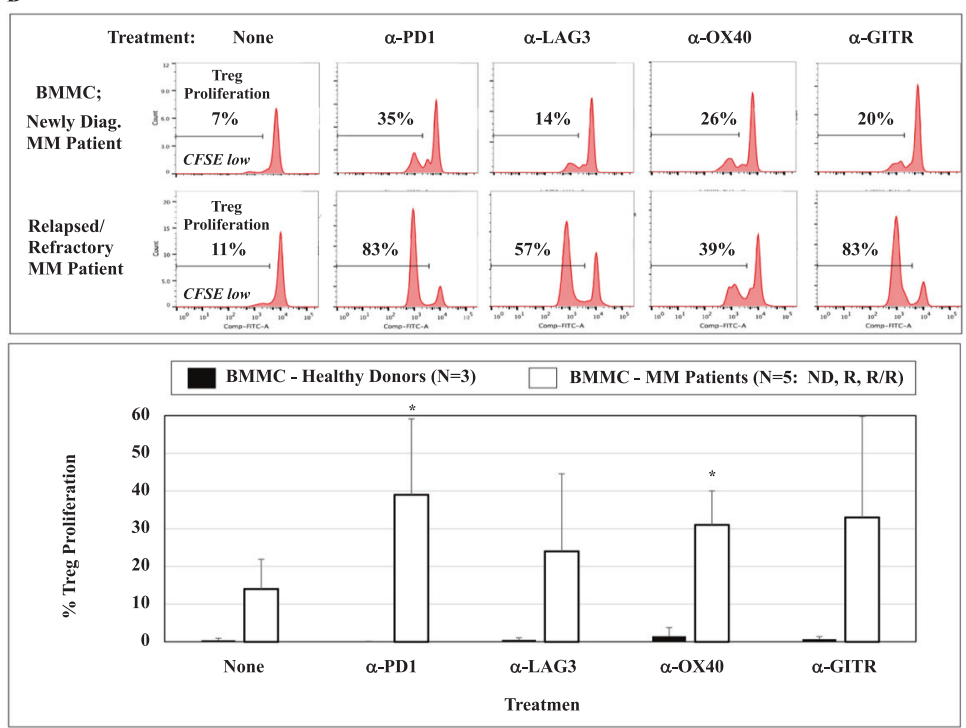

C

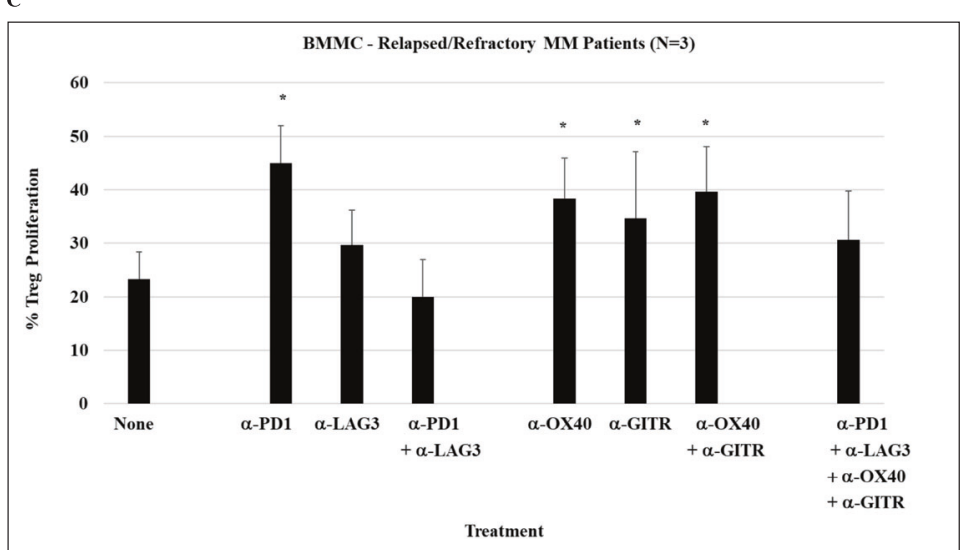

inhibitors, which was not detected in combination treatment with immune agonists; among various combination treatments evaluated, the lowest level of Treg proliferation was noted with anti-PD1 plus anti-LAG3. These results indicate a potential mechanism of immune resistance to checkpoint therapy whereby treatment with a checkpoint inhibitor 
Fig. 6 Impact of checkpoint inhibitor treatment on antiMM activities of XBP1/ CD138/CS1-specific CTL. HLA-A2-specific XBP1/CD138/ CS1-specific CTL $(N=5)$ were generated by four cycles of weekly stimulation of $\mathrm{CD}^{+}$

$\mathrm{T}$ cells with immunogenic XBP1/CD138/CS1 peptides and then examined for their phenotypic profile and functional activities against MM. A Upon fourth cycle of peptides stimulation, timedependent T-cell activation (CD69) and checkpoint (CTLA4, PD1, LAG3, VISTA, TIM3) upregulation were detected on MM-specific CTL. B, C Immune checkpoint treatment enhanced (anti-LAG3 $>$ anti-PD1) the poly-functional activities of antigen-specific $\mathrm{T}$ cells in response to HLA-A2matched U266 MM cells. B Induced proliferation of total $\mathrm{CD}^{+} \mathrm{CTL}$ as well as central memory, effector memory, $\mathrm{CD} 28^{+}$and $\mathrm{CD} 38^{+} \mathrm{CTL}$ subsets. C Increased CD107a ${ }^{+}$ degranulation and IFN- $\gamma$ production associated with a higher $\mathrm{CD}^{+}$CTL proliferation.

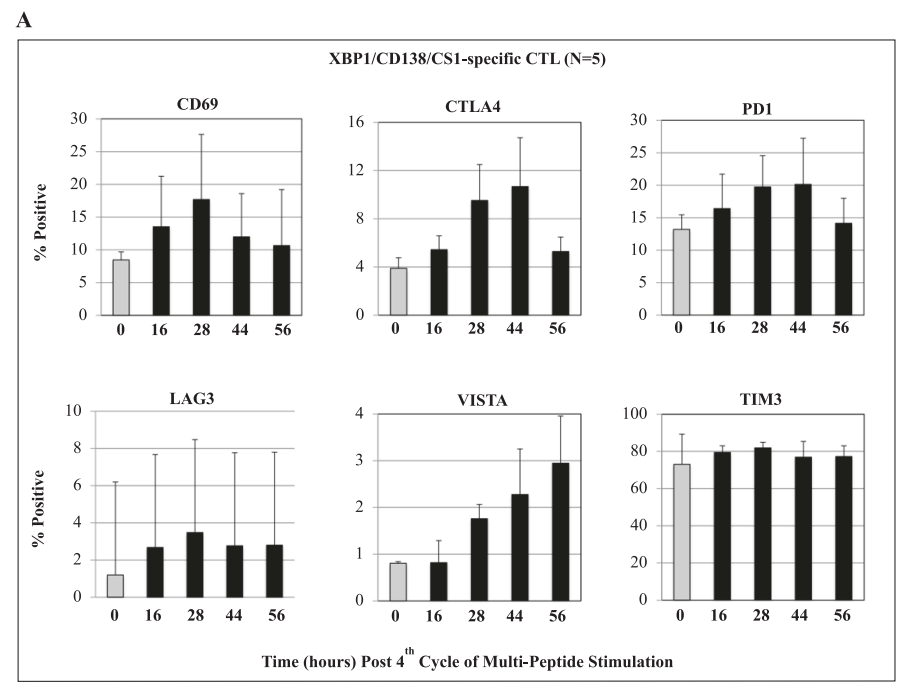

B
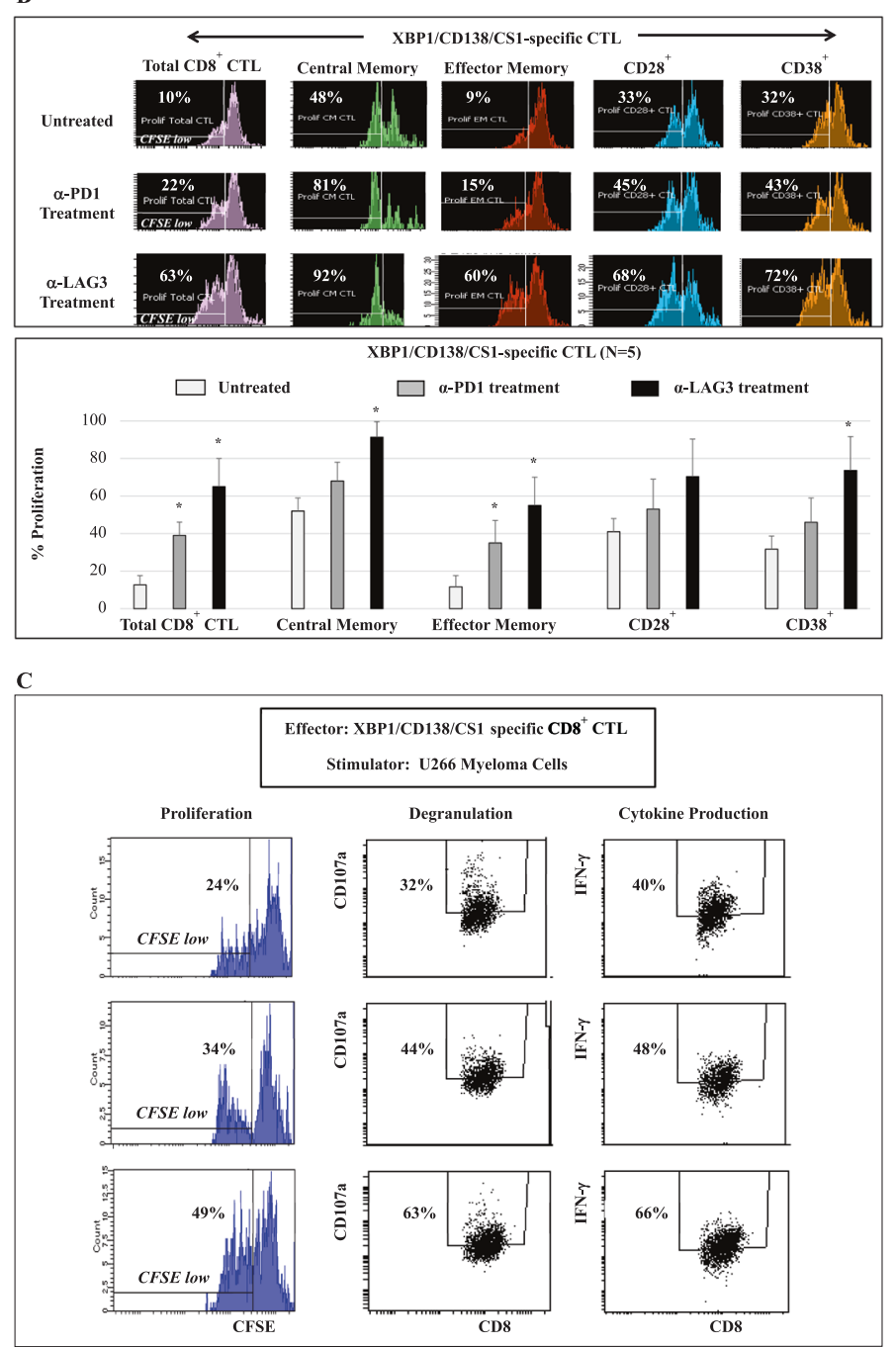

induces immune suppressive cells and nontargeted immune checkpoints in MM patients BMMC, suggesting the need for combination modulator treatment to overcome resistance to single agent immunotherapy approaches. 


\section{Increased functional anti-MM activity of XBP1/ CD138/CS1-specific CTL treated with anti-LAG3}

We next evaluated the functional significance of immune modulator therapy by examining its impact on anti-tumor activity of MM-specific CTL generated with HLA-A2 XBP1/CD138/CS1 peptides including heteroclitic XBP1 $\mathrm{US}_{184-192}$ (YISPWILAV), heteroclitic XBP1 SP $367-375$ (YLFPQLISV), native CD138 $260-268$ (GLVGLIFAV), and native $\mathrm{CS}_{239-247}$ (SLFVLGLFL), as described previously [14-17]. Phenotypic analyses after four cycles of weekly stimulation with peptides demonstrated time-dependent increased expression of CD69 activation marker and CTLA4, PD1, LAG3, and VISTA immune checkpoints on XBP1/CD138/CS1-specific CTL $(N=5)$ (Fig. 6A). In response to stimulation with HLA-A2 matched MM cells (U266), the central memory CD8 ${ }^{+} \mathrm{T}$-cell subset displayed the highest proliferation (48\%). Importantly, the XBP1/ CD138/CS1-CTL treated with clinical grade anti-LAG3 or anti-PD1 increased $(\alpha$-LAG3 $>\alpha-$ PD1) a significant $(* p<$ 0.05 ) proliferation of total $\mathrm{CD}^{+} \mathrm{T}$ cells as well as CM, EM, $\mathrm{CD} 28^{+}$, and $\mathrm{CD} 38^{+}$CTL subsets (Fig. 6B; histograms, bar graph $[N=5])$. The treatment with each checkpoint inhibitor also increased anti-tumor activities of XBP1/CD138/ CS1-CTL against MM cells, evidenced by increased CD107a degranulation and IFN- $\gamma$ production, with the highest anti-tumor activities induced by anti-LAG3 treatment (Fig. 6C). These results further support the ability of LAG3 blockade to augment anti-MM immune responses including antigen-specific memory CTL, their cytotoxic activities, and Thl cytokine production against tumor.

\section{Surface and intracellular expression of LAG3 ligands, GAL-3 and HLA-DP/DQ/DR, in CD138 ${ }^{+}$cells in $\mathrm{MM}$ patient $\mathrm{BMMC}$, and induction of specific $\mathrm{CD8}^{+}$ Tc proliferation by blocking GAL-3}

Having shown the functional significance of the LAG3 immune checkpoint in MM, we next assessed its ligands GAL-3 and HLA-DP/DQ/DR. The expression and role of each of LAG3 ligand was analyzed in BMMC from MM patients $(N=4$; newly diagnosed, relapsed, relapsed refractory). $\mathrm{CD} 138^{+} \mathrm{MM}$ cells demonstrated the expression of both GAL-3 and HLA-DP/DQ/DR, with greater expression evidenced by higher median fluorescence intensity intracellularly than on the cell surface (Fig. 7A). We next examined the functional significance of GAL-3 and HLA-DP/DQ/DR blockade in MM. Treatment of newly diagnosed MM patient BMMC with anti-GAL-3, but not with anti-HLA-DP/DQ/DR, induced proliferation of T cells, including $\mathrm{CD}^{+}$Tc cells (45\%: Patient \#1, 38\%: Patient \#2) and $\mathrm{CD}^{+}$Th cells (13\%: Patient \#1, 9\%: Patient \#2) (Fig. 7B). Additional analyses of BMMC from MM patients (newly diagnosed, relapsed, relapsed/refractory; $N=5$ ) showed a significant $(* p<0.05)$ increase in proliferation of $\mathrm{CD} 8^{+} \mathrm{Tc}$ and $\mathrm{CD} 4^{+}$Th cells $\left(\mathrm{CD}^{+}>\mathrm{CD} 4^{+}\right)$triggered by GAL-3 blockade (Fig. 7C). These results indicate the potential benefit of GAL-3 blockade in MM patients to induce $\mathrm{T}$ cells specific responses with a high level of $\mathrm{CD} 8^{+}$ $\mathrm{T}$ cells proliferation.

\section{Induction of MM-specific CD8 ${ }^{+} \mathrm{T}_{\text {-cell proliferation }}$ and anti-tumor activities by blocking GAL-3 on MM cells}

Finally, we examined the impact of inhibiting LAG3 ligand on MM-specific CTL activities. We first extended our analysis of GAL-3 and HLA-DP/DQ/DR to MM cell lines (MM1S, OPM2, RPMI8226, H929, U266, AMO1). Overall, a higher HLA-DP/DQ/DR surface and intracellular expression was detected than GAL-3 expression, whereas GAL-3 displayed a greater $\left({ }^{*} p<0.05\right)$ level of intracellular than cell surface expression $(N=3)$ (Fig. $8 \mathrm{~A})$. We further examined the functional impact of GAL-3 or HLA-DP/DQ/ DR blockade on the specific proliferation and anti-tumor activities of MM-specific CD8 ${ }^{+} \mathrm{CTL}$ against $\mathrm{MM}$ cells in an HLA-A2-specific manner. As shown in Fig. 8B, proliferation of HLA-A2 XBP1/CD138/CS1-specific CTL was enhanced in response to HLA-A2 ${ }^{+}$U266 MM cells upon the treatment with anti-GAL-3, but not with anti-HLA-DP/ $\mathrm{DQ} / \mathrm{DR}$, in an effector (CTL):target (MM cells)-dependent manner $(1: 1>1: 0.5>1: 0.25)$ (histograms, bar graphs $[N=$ 5]). Importantly, the specific blockade of GAL-3 in MM cells further increased proliferation of LAG3 expressing $\mathrm{XBP1/CD138/CS1-CTL} \mathrm{(Fig.} \mathrm{8C),} \mathrm{suggesting} \mathrm{an} \mathrm{alter-}$ native escape mechanism after anti-GAL-3 therapy in MM patients. Taken together, these results identify the functional relevance of blocking GAL-3 on MM cells as a means to enhance effector T-cell activities, and also provide the rationale for targeting GAL-3 (on MM tumor cells) in combination with LAG3 (on effector $\mathrm{T}$ cells) to further enhance MM-specific immune responses and anti-tumor activities.

\section{Discussion}

Understanding the biologic and immune sequelae of tumor cell interaction with accessory and immune cells in the tumor microenvironment is crucial for the development of successful cancer immunotherapies. Effective therapeutic strategies in MM may not only target tumor and tumorpromoting accessory cells, but also abrogate mechanisms mediating immunosuppression in the BM milieu [21-23]. Previously, our group has delineated the role of accessory cells (MDSC, plasmacytoid dendritic cells, Treg, 
Fig. 7 Expression and role of LAG3 ligands, GAL-3 and HLA-DP/DQ/DR, on CD138 ${ }^{+}$ cells in BMMC or PBMC from MM patients. Phenotype and functional characterization of LAG3 ligands, GAL-3 and HLA-DP/DQ/DR, were evaluated in MM patients' BMMC by flow cytometry. A GAL-3 and HLA-DP/DQ/DR were both expressed

(intracellular $>$ cell surface) in CD138 ${ }^{+}$tumor cells from MM patients $(N=4)$. B Treatment of MM patient BMMC with anti-

GAL-3, but not with anti-HLADP/DQ/DR, increased proliferation of $\mathrm{T}$ cells $\left(\mathrm{CD} 8^{+} \mathrm{Tc}>\mathrm{CD} 4^{+} \mathrm{Th}\right)$ in BMMC from MM patients (Patient \#1, Patient \#2). C GAL3 blockade induced an increased $(* p<0.05)$ proliferation of both $\mathrm{CD}^{+}{ }^{+} \mathrm{Tc}$ and $\mathrm{CD} 4^{+}$Th cells $\left(\mathrm{CD}^{+}>\mathrm{CD}^{+}\right)$in BMMC from MM patients $(N=5)$.
A
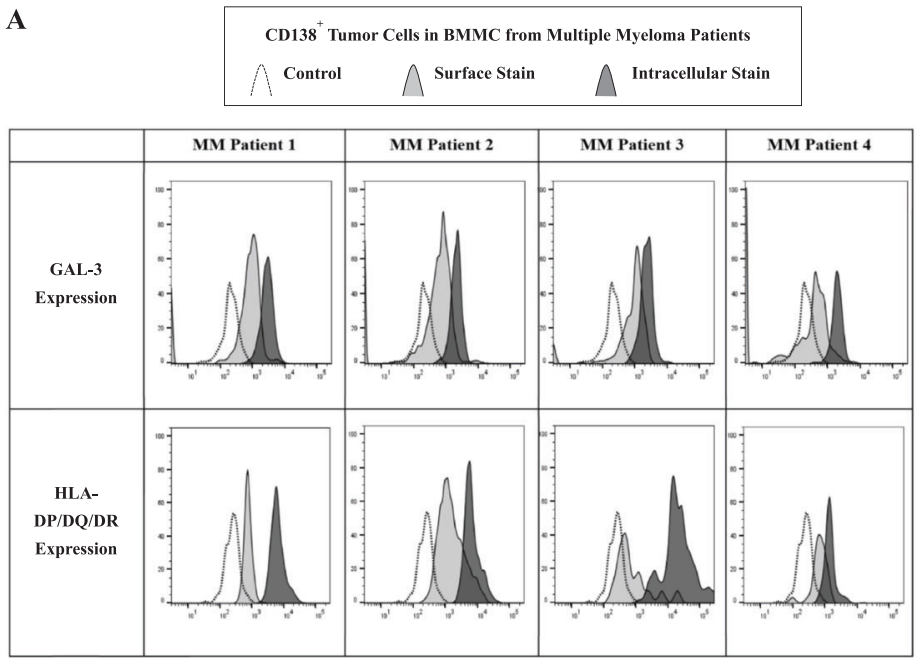

B

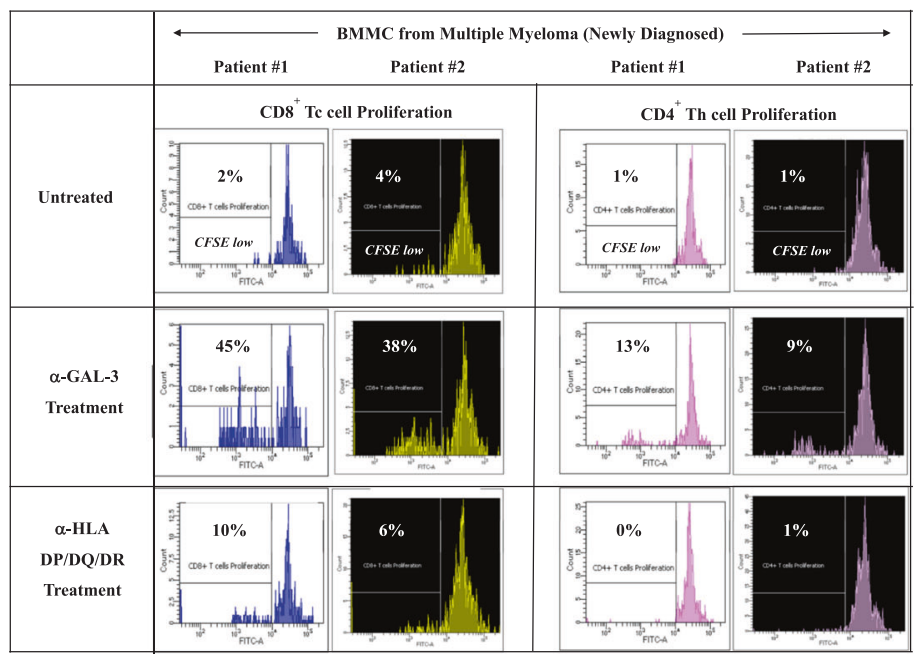

C

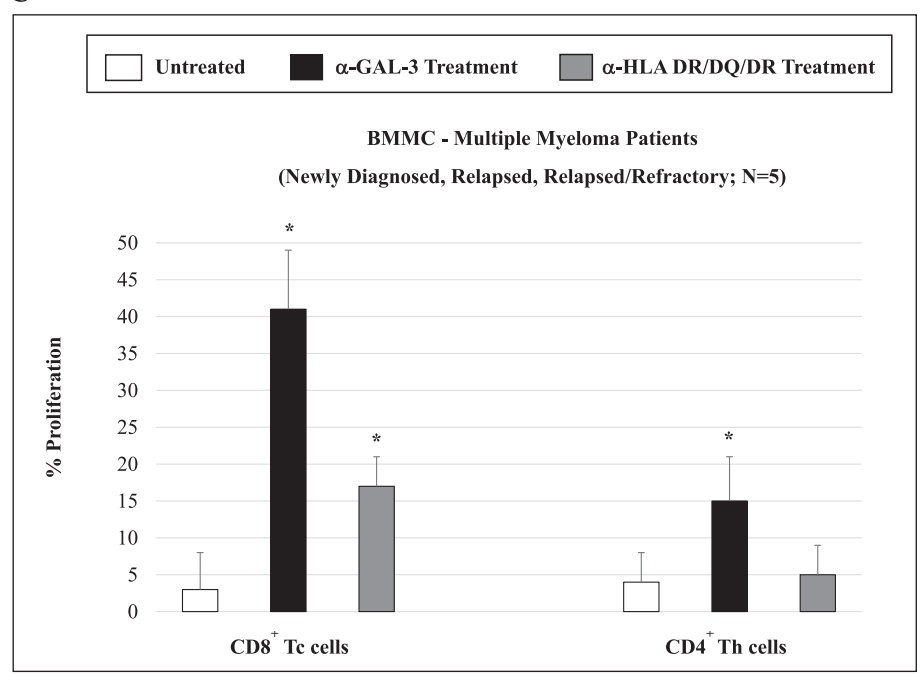


Fig. 8 Impact of inhibition of LAG3 ligands on proliferation and antitumor activities of MM-specific CTL. The role of the LAG3 ligands was analyzed using XBP1/CD138/CS1specific $\mathrm{CD}^{+}$CTL against MM cells. A GAL-3 and HLA-DP/ DQ/DR are both expressed in $\mathrm{CD} 138^{+}$tumor cells of MM cell lines $(N=3)$, with higher $(* p<0.05)$ intracellular than cell surface expression of GAL-3. B HLA-A2 ${ }^{+}$XBP1/CD138/ CS1-specific CTL $(N=5)$ demonstrated increased MMspecific $\mathrm{CD} 8^{+} \mathrm{CTL}$ proliferation in response to HLA-A2 ${ }^{+}$U266 MM cells treated with anti-

GAL-3, but not with anti-HLA$\mathrm{DP} / \mathrm{DQ} / \mathrm{DR}$, in a dose (U266 cells)-dependent manner (XBP1/ CD138/CS1-CTL: MM cells $=$ $1: 1>1: 0.5>1: 0.25)$.

C Proliferation of $\mathrm{LAG}^{+}$cells was triggered in XBP1/CD138/ CS1-specific CTL by stimulation with U266 treated with anti-GAL-3, but not with anti-HLA-DP/DQ/DR, in a dose (U266)-dependent manner (XBP1/CD138/CS1-CTL: MM cells $=1: 1>1: 0.5>1: 0.25>$ 1:0).
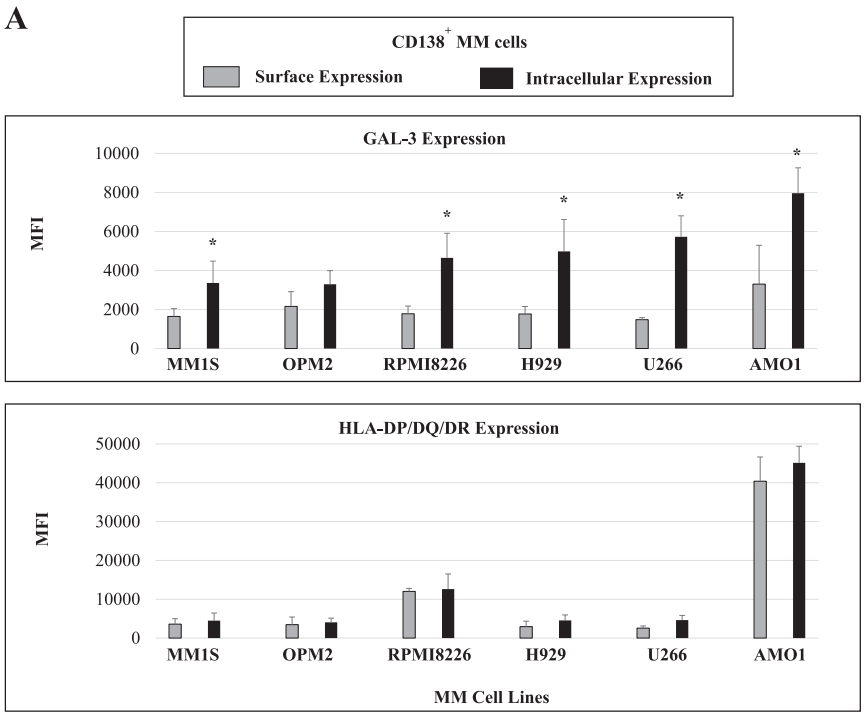

B
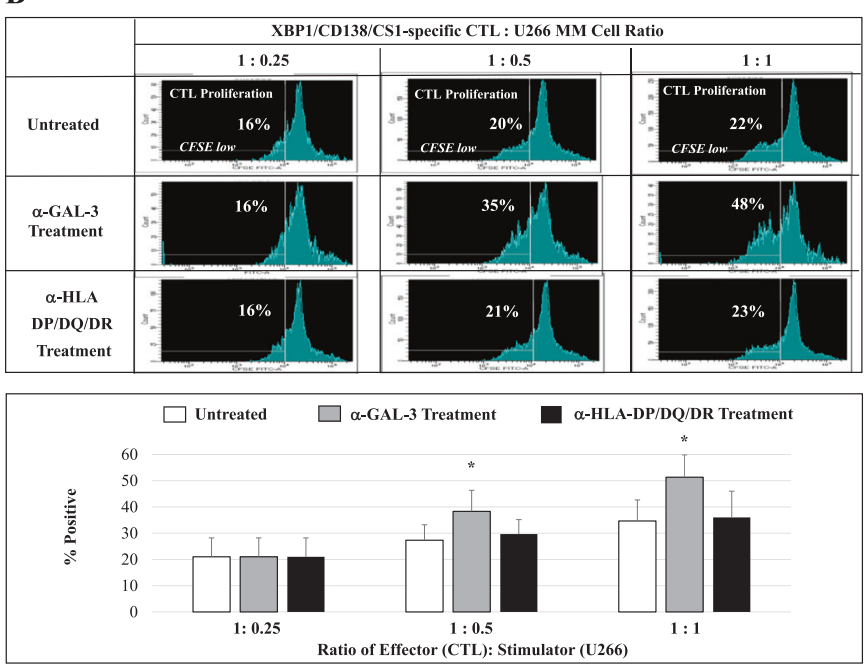

C

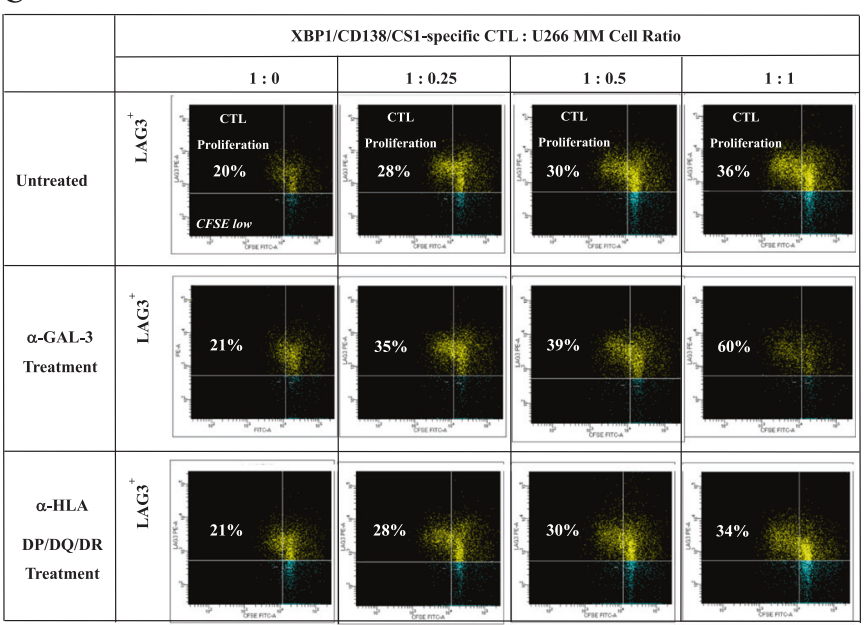


osteoclasts) in promoting tumor cell growth and survival and drug resistance, as well as conferring immunosuppression in MM [24-26]. In the current study, we further characterized the distribution, location, and expression levels of immune checkpoints not only on effector T cells, but also on MM cells and immune regulatory/suppressor cells in the BM and $\mathrm{PB}$ of patients with $\mathrm{MM}$ (newly diagnosed, relapsed, relapsed/refractory), premalignant diseases (MGUS, SMM), and healthy individuals. Our analyses revealed key differences in the frequency of cellular subsets (immune effector, regulatory/suppressor vs. tumor cells) and expression of immune checkpoints/agonists in patients with active MM compared to MGUS/SMM and healthy donors.

Since effective immunotherapy depends upon robust effector T-cell function [27-29], we first defined the presence and function of endogenous T-cell subsets in MM patient $\mathrm{BM}$ and $\mathrm{PB}$. Although proliferating $\mathrm{CD}^{+} \mathrm{T}$ cells in the presence of IL-2 or MM cell lysates expressed multiple immune modulators in these studies, the immune checkpoint LAG3 was most highly expressed on both proliferating $\mathrm{CD}^{+}{ }^{+} \mathrm{Th}$ and $\mathrm{CD} 8^{+} \mathrm{Tc}$ cells, and anti-LAG3 treatment most significantly enhanced their MM-specific immune responses. Our studies further demonstrated decreased effector $\mathrm{CD}^{+}$Th cells, increased Treg and Gtype MDSC, as well as upregulation of immune checkpoints on both effector/regulatory cells and patients CD138 tumor cells in MM, compared to patients with MGUS and SMM or healthy individuals. Of immune modulators profiled, LAG3 expression and impact of anti-LAG3 treatment was low on G-type MDSC and Tregs, suggesting that it will not enhance immunosuppression conferred by these accessory cells in the BM milieu. In evaluation of XBP1/CD138/ CS1 peptides-specific CTL with anti-MM activity, we confirmed that anti-LAG3 treatment induced enhanced proliferation of both CM and EM memory CTL subsets and their functional anti-MM activities including cytotoxicity and Th1-type cytokine production. Importantly, we also identified GAL-3, the ligand for LAG3, to be robustly expressed on $\mathrm{CD} 138^{+} \mathrm{MM}$ cells, and confirmed that antiGAL-3 treatment can similarly augment immune responses against MM cells in patient BM, as well as XBP1/CD138/ CS1 antigen-specific CTL. These studies identify and validate the potential blockade of LAG3/GAL-3 to enhance anti-tumor immune responses in MM.

Checkpoint blockade is a revolutionary cancer immunotherapy; however, a large proportion $(70-80 \%)$ of checkpoint inhibitor-treated cancer patients do not benefit due to either intrinsic or acquired resistance [30-33]. Multiple factors contribute to checkpoint blockade resistance including a lack of antigen-specific immune responses and/ or impaired infiltration of effector T cells to tumor sites [3439]. An important goal of our studies was to better elucidate potential mechanisms whereby immune inhibitory receptors and ligands regulate innate and adaptive immunity in MM, and specifically delineate potential mechanisms of resistance to checkpoint blockade in MM. We demonstrated a direct beneficial impact of checkpoint inhibitor treatment on T-cell functional activity in BM cells from MM patients. Specifically, checkpoint inhibitor (especially anti-LAG3) treatment significantly increased T-cell responses in BMMC/PBMC from MM patients compared with healthy donors. Along with identifying the potential functional role of checkpoint inhibitors, we also examined the impact of immune agonists such as OX40 (CD134) and GITR (CD357) to activate costimulatory molecules on effector cells and thereby enhance their immune responses [36, 37]. In both MM patient BMMC and PBMC, immune agonist treatment enhanced immune responses and T-cell proliferation. Importantly, our finding of higher intracellular than cell surface checkpoint expression on $\mathrm{CD}^{+} \mathrm{T}$ cells and $\mathrm{CD} 138^{+} \mathrm{MM}$ cells in patient BMMC suggests that high intracellular levels of checkpoints may provide a continuous source of checkpoint molecules for translocation to the cell surface, thereby maintaining ongoing checkpoint-driven immune resistance in MM. Moreover, our studies show that treatment of MM patient BMMC with one checkpoint inhibitor can upregulate expression of another checkpoint, as well as expansion of regulatory and suppressor cells, in addition to effector $\mathrm{CD}^{+} \mathrm{T}$ cells. Taken together, these data identify alternative mechanisms of immune resistance induced by checkpoint inhibitors and immune agonists in MM.

Among the clinical grade checkpoint inhibitors and immune agonists evaluated in these studies, anti-PD1 treatment induced the highest level of $\mathrm{CD}^{+}{ }^{+}$Treg expansion and upregulation of other immune checkpoints. Importantly, antiLAG3 treatment induced the most robust effector T-cell proliferation, while inducing the lowest level of induction of other checkpoints and Treg expansion. In addition, we detected a higher intracellular expression of LAG3 compared to PD1 in MM patient $(N=5)$ BMMC. Based on these findings, we suggest that LAG3 blockade in MM may be more effective than PD1 blockade, with a lower induction of alternative checkpoint molecules and a higher induction of effector T-cell proliferation and response. These results are of particular relevance, given recent toxicity concerns observed when combining pembrolizumab (anti-PD1) with immunomodulatory drugs lenalidomide or pomalidomide or with daratumumab (anti-CD38) in recent clinical trials for relapsed MM patients [40-42]. Considering the robust LAG3 expression in the tumor microenvironment and its correlation with poor prognosis in MM and other cancers [23, 43-46], targeting the LAG3-specific inhibitory pathway may enhance anti-MM immunity and have a more favorable therapeutic index. Importantly, among the clinical grade checkpoint inhibitors and immune agonists evaluated in these studies, 
anti-LAG3 treatment significantly enhanced the proliferation of MM-specific effector cells and their functional activities in response to MM. Based on these results, we propose that antiLAG3 may effectively overcome immunosuppression in the tumor microenvironment and be used, alone or in combination with other immune therapies such as MM-specific vaccination, to enhance generation and maintenance of antigenspecific memory CTL function against tumor.

Characterization of inhibitory checkpoint ligands as well as mechanisms of their interaction and consequent effector T-cell suppression are critical to better design clinical studies and achieve long-term anti-tumor immunity in MM patients. Pharmacological blockade of PD1 or PD-L1 has been at the forefront of immunotherapy for various cancers, as it reinvigorates exhausted $\mathrm{T}$ cells in the tumor microenvironment, thereby facilitating robust anti-tumor immune responses. However, up to $50 \%$ of patients with PD-L1 positive tumors show acquired resistance or relapse after an initial response to PD1/PD-L1 blockade [35, 47-49], highlighting the need to target alternative pathways of inhibitory checkpoint receptor/ligand interaction to improve clinical outcomes. To address this concern and in the context of our promising anti-LAG3 data in MM, we went on to evaluate expression of LAG3 ligands, GAL-3 and HLA-DP/ $\mathrm{DQ} / \mathrm{DR}$, on $\mathrm{CD} 138^{+} \mathrm{MM}$ cells in patient BMMC and MM cell lines, identifying them as promising therapeutic targets. We found that blockade of GAL-3, but not HLA-DP/DQ/ $\mathrm{DR}$, enhanced proliferation of both $\mathrm{CD} 4^{+} \mathrm{Th}$ and $\mathrm{CD} 8^{+} \mathrm{Tc}$ cell subsets in BMMC from MM patients, independent of the cell surface or intracellular expression levels of the two respective ligands on primary $\mathrm{CD} 138^{+} \mathrm{MM}$ cells. Thus, we propose that checkpoint ligands expression level itself might not be the only factor, which influence effector T-cell function and proliferation. The expression level and specificity/affinity between checkpoint receptor (LAG3) on the patients' $T$ cells and the checkpoint ligands (GAL-3, HLA$\mathrm{DP} / \mathrm{DQ} / \mathrm{DR}$ ) on patients' tumor cells are a critical consideration impacting the functional sequelae of their interaction. Recently, Kundapura and Ramagopal demonstrated that the $\mathrm{CC}^{\prime}$ loop of $\mathrm{IgV}$ domains of the immune checkpoint receptors, a loop which is distinct from CDRs of antibodies, plays a pivotal role in receptor: ligand affinity modulation [50]. They proposed that a $\sim 5$ amino acid residue long $\mathrm{CC}^{\prime}$ loop in a $\sim 120$ residue protein makes a significant number of hydrophobic and polar interactions with its cognate checkpoint ligand and suggested that the $\mathrm{CC}^{\prime}$ loop might be a hotspot for checkpoint receptor modification that enhance their affinity for ligand interaction. In addition, we propose that the interaction between receptor and ligand can be influenced by the unique T-cell receptor repertoire of each individual, resulting in variable levels or profiles of T-cell or CTL functional responses and proliferation. Taken together, our results in MM are consistent with previous reports on the role of GAL-3 as a key regulator of cell adhesion and inflammation in cancer [51-56]; it negatively regulates $\mathrm{T}$-cell function and proliferation through interaction with LAG3, especially on $\mathrm{CD} 8^{+} \mathrm{CTL}$, possibly by reducing the affinity of the $\mathrm{T}$-cell receptor and its internalization. Importantly, we observed increased MM-specific $\mathrm{CD}^{+} \mathrm{Tc}$ cells expansion and selective anti-MM immune activities after anti-GAL-3 treatment of both MM patient BMMC and XBP1/CD138/CS1-specific CTL. These findings further indicate the potential role for LAG3 and/or GAL-3 inhibition, alone and with XBP1/CD138/CS1 peptide vaccination, to augment $\mathrm{MM}$-specific memory $\mathrm{CD} 8^{+}$ CTL anti-tumor activities against MM.

In summary, we have identified a key immunosuppressive role for LAG3 and its ligand GAL-3 in regulating innate and adaptive immunity in $\mathrm{MM}$ and provide the rationale for targeting LAG3/GAL-3, alone and in combination immunotherapeutic approaches, to improve patient outcome in MM.

Acknowledgements The grant support for this investigation was provided by National Institutes of Health Specialized Programs of Research Excellence (SPORE) grants P50100707, R01CA207237, PO1 CA155258, and RO1 CA050947. This work was supported in part by Dr. Miriam and Sheldon G. Adelson Medical Research Foundation and the Riney Family Multiple Myeloma Initiative, as well as by Bristol Myers Squibb.

\section{Compliance with ethical standards}

Conflict of interest KCA is an advisor/consultant for Bristol Myers Squibb, Janssen, Pfizer, Amgen, AstraZeneca, OncoPep, and Precision Biosciences, and is a scientific founder of $\mathrm{C} 4$ therapeutics and OncoPep. NCM is in consultant/advisory board for Bristol Myers Squibb, Takeda, Janssen, AbbVie, Karyopharm, Adaptive, OncoPep, and Amgen, and is a scientific founder of OncoPep. PGR has research support from Bristol Myers Squibb, research support and honoraria (advisory committee member) from Oncopeptides, Celgene, Takeda, and Karyopharm, and honoraria (advisory committee member) from Janssen, Sanofi, and Secura Bio outside the submitted work. JB is in advisory board and a scientific founder of OncoPep. No relevant conflicts of interest were disclosed by the other authors.

Publisher's note Springer Nature remains neutral with regard to jurisdictional claims in published maps and institutional affiliations.

Open Access This article is licensed under a Creative Commons Attribution 4.0 International License, which permits use, sharing, adaptation, distribution and reproduction in any medium or format, as long as you give appropriate credit to the original author(s) and the source, provide a link to the Creative Commons license, and indicate if changes were made. The images or other third party material in this article are included in the article's Creative Commons license, unless indicated otherwise in a credit line to the material. If material is not included in the article's Creative Commons license and your intended use is not permitted by statutory regulation or exceeds the permitted use, you will need to obtain permission directly from the copyright holder. To view a copy of this license, visit http://creativecommons. org/licenses/by/4.0/. 


\section{References}

1. Lomas OC, Tahri S, Ghobrial IM. The microenvironment in myeloma. Curr Opin Oncol. 2020;32:170-5.

2. Kawano Y, Roccaro AM, Ghobrial IM, Azzi J. Multiple myeloma and the immune microenvironment. Curr Cancer Drug Targets. 2017;17:806-18.

3. Anderson KC. Insights into the management of older patients with multiple myeloma. Clin Adv Hematol Oncol. 2019;17:390-2.

4. Finn OJ. A believer's overview of cancer immunosurveillance and immunotherapy. J Immunol. 2018;200:385-91.

5. Dyck L, Mills KHG. Immune checkpoints and their inhibition in cancer and infectious diseases. Eur J Immunol. 2017;47:s765-79.

6. Raza A, Merhi M, Inchakalody VP, Krishnankutty R, Relecom A, Uddin $\mathrm{S}$, et al. Unleashing the immune response to NY-ESO-1 cancer testis antigen as a potential target for cancer immunotherapy. J Transl Med. 2020;18:140-50.

7. Schirrmacher V. Cancer vaccines and oncolytic viruses exert profoundly lower side effects in cancer patients than other systemic therapies: a comparative analysis. Biomedicines. 2020;8:61-82.

8. Kremenovic M, Schenk M, Lee DJ. Clinical and molecular insights into BCG immunotherapy for melanoma. J Intern Med. 2020. https://doi.org/10.1111/joim.13037.

9. Im SJ, Ha SJ. Re-defining T-cell exhaustion: subset, function, and regulation. Immune Netw. 2020;20:e2.

10. Gálvez-Cancino F, López E, Menares E, Díaz X, Flores C, Cáceres $\mathrm{P}$, et al. Vaccination-induced skin-resident memory $\mathrm{CD} 8+\mathrm{T}$ cells mediate strong protection against cutaneous melanoma. Oncoimmunology. 2018;7:e1442163.

11. Bae J, Hideshima T, Zhang GL, Zhou J, Keskin DB, Munshi NC, et al. Identification and characterization of HLA-A24-specific XBP1, CD138 (Syndecan-1) and CS1 (SLAMF7) peptides inducing antigens-specific memory cytotoxic $\mathrm{T}$ lymphocytes targeting multiple myeloma. Leukemia. 2018;32:752-64.

12. Coxon AT, Johanns TM, Dunn GP. An innovative immunotherapy vaccine with combination checkpoint blockade as a first line treatment for glioblastoma in the context of current treatments. Mo Med. 2020;117:45-49.

13. Shibata T, Lieblong BJ, Sasagawa T, Nakagawa M. The promise of combining cancer vaccine and checkpoint blockade for treating HPV-related cancer. Cancer Treat Rev. 2019;78:8-16.

14. Bae J, Carrasco R, Lee AH, Prabhala R, Tai YT, Anderson KC, et al. Identification of novel myeloma-specific XBP1 peptides able to generate cytotoxic $\mathrm{T}$ lymphocytes: potential therapeutic application in multiple myeloma. Leukemia. 2011;25:1610-9.

15. Bae J, Tai Y, Anderson KC, Munshi N. Novel epitope evoking CD138 antigen-specific cytotoxic T lymphocytes targeting multiple myeloma and other plasma cell disorders. Br J Haematol. 2011;155:349-61.

16. Bae J, Song W, Smith R, Daley J, Tai YT, Anderson KC, et al. A novel immunogenic CS1-specific peptide inducing antigenspecific cytotoxic $\mathrm{T}$ lymphocytes targeting multiple myeloma. Br J Haematol. 2012;157:687-701.

17. Bae J, Smith R, Daley J, Mimura N, Tai YT, Anderson KC, et al. Myeloma-specific multiple peptides able to generate cytotoxic $\mathrm{T}$ lymphocytes: a potential therapeutic application in multiple myeloma and other plasma cell disorders. Clin Cancer Res. 2012;18:4850-60.

18. Nooka AJ, Wang M, Yee AJ, Kaufman J, Bae J, Peterkin D, et al. Assessment of safety and immunogenicity of PVX-410 vaccine with or without lenalidomide in patients with smoldering multiple myeloma: a nonrandomized clinical trial. JAMA Oncol. 2018;4: e183267.

19. Karyampudi L, Lamichhane P, Scheid AD, Kalli KR, Shreeder B, Krempski JW, et al. Accumulation of memory precursor CD8
$\mathrm{T}$ cells in regressing tumors following combination therapy with vaccine and anti-PD1 antibody. Cancer Res. 2014;74: 2974-85.

20. Le DT, Lutz E, Uram JN, Sugar EA, Onners B, Solt S, et al. Evaluation of ipilimumab in combination with allogeneic pancreatic tumor cells transfected with a GM-CSF gene in previously treated pancreatic cancer. J Immunother. 2013;36:382-9.

21. Solinas C, Migliori E, De Silva P, Willard-Gallo K. LAG3: the biological processes that motivate targeting this immune checkpoint molecule in human cancer. Cancers. 2019;11:E1213.

22. Doroshow DB, Sanmamed MF, Hastings K, Politi K, Rimm DL, Chen L, et al. Immunotherapy in non-small cell lung cancer: facts and hopes. Clin Cancer Res. 2019;25:4592-602.

23. Esteva FJ, Hubbard-Lucey VM, Tang J, Pusztai L. Immunotherapy and targeted therapy combinations in metastatic breast cancer. Lancet Oncol. 2019;20:175-86.

24. Prabhala RH, Neri P, Bae JE, Tassone P, Shammas MA, Allam $\mathrm{CK}$, et al. Dysfunctional $\mathrm{T}$ regulatory cells in multiple myeloma. Blood. 2006;107:301-14.

25. Chauhan D, Singh AV, Brahmandam M, Carrasco R, Bandi M, Hideshima $\mathrm{T}$, et al. Functional interaction of plasmacytoid dendritic cells with multiple myeloma cells: a therapeutic target. Cancer Cell. 2009;16:309-23.

26. Görgün GT, Whitehill G, Anderson JL, Hideshima T, Maguire C, Laubach J, et al. Tumor-promoting immune-suppressive myeloidderived suppressor cells in the multiple myeloma microenvironment in humans. Blood. 2013;121:2975-87.

27. van der Leun AM, Thommen DS, Schumacher TN. CD8(+) T cell states in human cancer: insights from single-cell analysis. Nat Rev Cancer. 2020;20:218-32.

28. Hashimoto M, Kamphorst AO, Im SJ, Kissick HT, Pillai RN, Ramalingam SS, et al. CD8 T cell exhaustion in chronic infection and cancer: opportunities for interventions. Annu Rev Med. 2018;69:301-18.

29. Thommen DS, Schumacher TN. T cell dysfunction in cancer. Cancer Cell. 2018;33:547-62.

30. Park R, Lopes L, Cristancho CR, Riano IM, Saeed A. Treatmentrelated adverse events of combination immune checkpoint inhibitors: systematic review and meta-analysis. Front Oncol. 2020;10:258.

31. Gremese E, Alivernini S, Ferraccioli ES, Ferraccioli G. Checkpoint inhibitors (CPI) and autoimmune chronic inflammatory diseases (ACIDs): tolerance and loss of tolerance in the occurrence of immuno-rheumatologic manifestations. Clin Immunol. 2020;214:108395.

32. Kumar P, Saini S, Prabhakar BS. Cancer immunotherapy with check point inhibitor can cause autoimmune adverse events due to loss of Treg homeostasis. Semin Cancer Biol. 2020;64:29-35.

33. Porcu M, De Silva P, Solinas C, Battaglia A, Schena M, Scartozzi M, et al. Immunotherapy associated pulmonary toxicity: biology behind clinical and radiological features. Cancers. 2019;11:305-20.

34. Mimura K, Kono K. Therapeutic cancer vaccine and immune checkpoint inhibitor. Gan Kagaku Ryoho. 2017;44:733-6.

35. Bai J, Gao Z, Li X, Dong L, Han W, Nie J. Regulation of PD1/ PD-L1 pathway and resistance to PD1/PD-L1 blockade. Oncotarget. 2017;8:110693-707.

36. Pham Minh N, Murata S, Kitamura N, Uek T, Kojima M, Miyake $\mathrm{T}$, et al. In vivo anti-tumor function of tumor antigen-specific CTLs generated in the presence of OX40 co-stimulation in vitro. Int J Cancer. 2018;142:2335-43.

37. Dushyanthen S, Teo ZL, Caramia F, Savas P, Mintoff CP, Virassamy B, et al. Agonist immunotherapy restores $\mathrm{T}$ cell function following MEK inhibition improving efficacy in breast cancer. Nat Commun. 2017;8:606. 
38. Kleponis J, Skelton R, Zheng L. Fueling the engine and releasing the break: combinational therapy of cancer vaccines and immune checkpoint inhibitors. Cancer Biol Med. 2015;12:201-8.

39. van der Burg SH, Arens R, Ossendorp F, van Hall T, Melief CJ. Vaccines for established cancer: overcoming the challenges posed by immune evasion. Nat Rev Cancer. 2016;16:219-33.

40. Chim CS, Kumar SK, Orlowski RZ, Cook G, Richardson PG, Gertz MA, et al. Management of relapsed and refractory multiple myeloma: novel agents, antibodies, immunotherapies and beyond. Leukemia. 2018;32:252-62.

41. Costa R, Costa RB, Talamantes SM, Helenoswki I, Carneiro BA, Chae YK, et al. Analyses of selected safety endpoints in phase 1 and late-phase clinical trials of anti-PD1 and PD-L1 inhibitors: prediction of immune-related toxicities. Oncotarget. 2017;8:67782-9.

42. Vanpouille-Box C, Lhuillier C, Bezu L, Aranda F, Yamazaki T, Kepp O, et al. Trial watch: immune checkpoint blockers for cancer therapy. Oncoimmunology. 2017;6:e1373237.

43. Zhou LJ, Tedder TF. Human blood dendritic cells selectively express CD83, a member of the immunoglobulin superfamily. J Immunol. 1995;154:3821-35.

44. Andrews LP, Marciscano AE, Drake CG, Vignali DA. LAG3 (CD223) as a cancer immunotherapy target. Immunol Rev. 2017;276:80-96.

45. Klümper N, Ralser DJ, Bawden EG, Landsberg J, Zarbl R, Kristiansen G, et al. LAG3 (LAG3, CD223) DNA methylation correlates with LAG3 expression by tumor and immune cells, immune cell infiltration, and overall survival in clear cell renal cell carcinoma. J Immunother Cancer. 2020;8:e00552.

46. Lucas F, Pennell M, Huang Y, Benson DM, Efebera YA, Chaudhry M, et al. T-cell transcriptional profiling and immunophenotyping uncover LAG3 as a potential significant target of immune modulation in multiple myeloma. Biol Blood Marrow Transpl. 2020;26:7-15.

47. Kato S, Okamura R, Kumaki Y, Sadakatsu Ikeda S, Nikanjam M, Eskander R, et al. Expression of TIM3/VISTA checkpoints and the CD68 macrophage-associated marker correlates with anti-
PD1/PDL1 resistance: implications of immunogram heterogeneity. Oncoimmunology. 2020;9:1708065.

48. Benci JL, Johnson LR, Choa R, Xu Y, Qiu J, Zhou Z, et al. Opposing functions of interferon coordinate adaptive and innate immune responses to cancer immune checkpoint blockade. Cell. 2019;178:933-48.

49. Herbst RS, Soria JC, Kowanetz M, Fine GD, Hamid O, Gordon MS, et al. Predictive correlates of response to the anti-PD-L1 antibody MPDL3280A in cancer patients. Nature. 2014;515:563-7.

50. Kundapura SV, Ramagopal UA. The CC' loop of IgV domains of the immune checkpoint receptors, plays a key role in receptor: ligand affinity modulation. Sci Rep. 2019;9:19191.

51. Mirandola L, Yu Y, Chui K, Jenkins MR, Cobos E, John CM, et al. Galectin-3C inhibits tumor growth and increases the anticancer activity of bortezomib in a murine model of human multiple myeloma. PLoS ONE. 2011;6:e21811.

52. Ruvolo PP. Galectin 3 as a guardian of the tumor microenvironment. Biochim Biophys Acta. 2016;1863:427-37.

53. Kouo T, Huang L, Pucsek AB, Cao M, Solt S, Armstrong T, et al. Galectin-3 shapes anti-tumor immune responses by suppressing CD8 + T cells via LAG3 and inhibiting expansion of plasmacytoid dendritic cells. Cancer Immunol Res. 2015;3:412-23.

54. Demotte N, Wieers G, Van Der Smissen P, Moser M, Schmidt C, Thielemans $\mathrm{K}$, et al. A galectin-3 ligand corrects the impaired function of human CD4 and CD8 tumor-infiltrating lymphocytes and favors tumor rejection in mice. Cancer Res. 2010;70:7476-88.

55. Fukumori T, Takenaka Y, Yoshii T, Kim HC, Hogan V, Inohara $\mathrm{H}$, et al. CD29 and CD7 mediate galectin-3-induced type II T-cell apoptosis. Cancer Res. 2003;63:8302-11.

56. Chen HY, Fermin A, Vardhana S, Weng IC, Lo KFR, Chang EY, et al. Galectin-3 negatively regulates TCR-mediated CD4+T-cell activation at the immunological synapse. Proc Natl Acad Sci USA. 2009;106:14496-501. 